\title{
Low-Noise Blade Design Optimization for a Transonic Fan Using Adjoint-Based Approach
}

\author{
L. Wu*, A. G. Wilson ${ }^{\dagger}$, and J. W. Kim ${ }^{\ddagger}$ \\ University of Southampton, Southampton, SO17 1BJ, United Kingdom \\ D. Radford ${ }^{\S}$ and S. Shahpar ${ }^{\text {II }}$ \\ Rolls-Royce plc., Derby, DE24 8BJ, United Kingdom
}

\begin{abstract}
The target of reducing the environmental footprint of the aviation industry has continually driven the need to design more efficient and quieter aircraft engines. In this paper, the aeroacoustic adjoint formulization for low-shock tone noise fan blade design is first proposed and combined with the aerodynamic adjoint analysis to develop an adjoint-based, multi-objective design optimization process for transonic fan blade design. High-fidelity numerical simulations are employed in the optimization loop to predict aeroacoustic and aerodynamic objectives and gradients. Aeroacoustic and aerodynamic design optimizations of an industrial transonic research fan are conducted using the proposed adjoint-based approach, demonstrating that the noise performance and the efficiency of the fan can be improved simultaneously. Results indicate that the significant reduction in the sound power level of the fan shock-associated tone noise is achieved by a compound leading-edge sweep pattern generated by the optimizations. Flow fields and acoustic results of the optimized blades are then analyzed to understand the noise reduction mechanism.
\end{abstract}

\section{Introduction}

$\mathrm{R}$ EDUCING noise and carbon emissions of aircraft engines is an inevitable task for the industry to achieve ambitious environmental targets, and therefore, requires continued efforts in design optimizations of all components. As a key component of the modern, high bypass-ratio turbofan engine, the fan contributes to the vast majority of the total thrust and its noise is the dominant one of all the engine noise sources especially during take-off and approach. Although acoustic liners have been widely used in the engine nacelle to mitigate fan noise propagation, further applications of this

\footnotetext{
Presented as Paper 2021-3055 at the virtual 2021 AIAA AVIATION Forum and Exposition, 2-6, August 2021.

Copyright $\odot 2021$ by the authors and Rolls-Royce plc. Member.

* Research Fellow, Rolls-Royce University Technology Centre in Propulsion Systems Noise, Institute of Sound and Vibration Research, AIAA

$\dagger$ Professor, Rolls-Royce University Technology Centre in Propulsion Systems Noise, Institute of Sound and Vibration Research.

†Professor, Rolls-Royce University Technology Centre in Propulsion Systems Noise, Institute of Sound and Vibration Research, AIAA Senior Member.

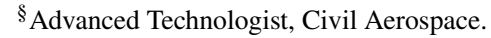

IIEngineering Fellow - Aerothermal Design System, Innovation Hub, Future Methods, Full Fellow of AIAA.
} 
technology are limited by the associated weight and drag impact. In this context, the development of low-noise blade design methods to reduce the fan noise source level at the design phase is of great need.

Historically, aerodynamic design has been at the core position of the fan blade design process, in which multiple performance parameters such as total pressure ratio, efficiency, stall margin, etc., are considered. However, the complexity of the real-life design task requires multi-objective (MOO) and multi-disciplinary (MDO) optimization approaches to be adopted to design the fan blade. Recent developments of computing power have enabled the application of high-fidelity numerical simulations in the design optimization process for detailed shape design. Many previous studies on the fan blade MDO mainly focused on the aerodynamic-mechanical interactions [1, 2], while the aeroacoustic design optimization for the fan blade received less attention. Wilson et al. [3] developed an MDO process to design a low tone noise transonic fan blade. The mechanical stresses of the blade were assessed to produce a constraint, and an aerodynamic redesign process was embedded in the low-noise optimization loop to meet the target aerodynamic duty. The final blade design was built and tested in a fan stage rig and was found to achieve significant shock-associated tone noise reduction. Kim et al. [4] performed an MOO on a subsonic axial-flow fan to enhance the aerodynamic and aeroacoustic performance using evolutionary algorithms coupled with surrogate models. In the present paper, special attention is paid to the aeroacoustic-aerodynamic design optimization of a transonic fan blade. The aim is to reduce the shock-associated tone noise of the fan and to improve its aerodynamic performance simultaneously.

For a fan operating in the transonic regime, shocks and expansion waves are generated close to the blade tip region. This "rotor-locked" field is propagated upstream along the intake duct because of the rotation. Strong buzz-saw tones at harmonics of the shaft rotation frequency, i.e. engine orders (EO), can be observed in the far-field due to the cut-on nature of the corresponding rotating modes at supersonic relative speeds [5]. Theoretically, a fan consisting of identical blades and subjected to a circumferentially uniform inflow would generate tones only at harmonics of the blade passing frequency (BPF). This ideal scenario greatly reduces the computational cost for simulating the shock-associated tone noise generation because a single-passage numerical model is sufficient. Therefore, the present paper takes the rotor-alone tones at BPF harmonics as the aeroacoustic objective in the formulation of the fan blade optimization problem. In practice, other EO tones, that are generated by minor blade-to-blade variations of manufacturing and installation, can be expected to broadly reduce alongside the rotor-alone tones at BPF harmonics [3].

Optimization algorithms generally employed in turbomachinery design can be classified into derivative-free methods and gradient-based methods [6]. A gradient-based search of the design space of a large-scale optimization problem is often more efficient than a global search using derivative-free methods, especially when boosted by the power of the adjoint approach [7, 8] for gradient computations, although global optimality cannot be guaranteed. For the steady computational fluid dynamics (CFD) solvers in turbomachinery, the discrete adjoint method [9] is often preferred. In this approach, the adjoint operator is obtained by 1) linearizing the discrete non-linear flow equations, and 2) transposing the linear operator of the discrete linear flow equations. The right-hand-side (RHS) term of the adjoint equations is directly 
obtained by linearizing the objective/constraint function of interest. The implementation of the discrete adjoint approach can be greatly facilitated by using automatic differentiation [10]. The discrete adjoint method has been successfully applied in the aerodynamic design optimization for turbomachinery [11, 12]. In this paper, discrete adjoint equations for the shock-associated tone noise of transonic fans are first developed. Then a two-disciplinary (i.e. aeroacoustics and aerodynamics) design optimization of a transonic research fan is carried out using the adjoint gradient-based method.

The remainder of this paper is organized as follows. Section $\Pi$ describes the discrete adjoint formulization for the low-shock tone noise blade design, and the numerical solver used for the present study. In Sec. III], the case setup for a transonic research fan is introduced and a mesh dependence study is conducted. Then both the nonlinear CFD simulation and the adjoint simulations are validated. In Sec. IV the adjoint-based, aeroacoustic and aerodynamic design optimizations are performed and the results are analyzed to discuss the mechanisms responsible for performance improvements. Finally, concluding remarks are made in Sec.V.

\section{Methodology and Numerical Solver}

The "rotor-locked" nature of the shock-associated tone noise permits the aeroacoustic and aerodynamic performance of the transonic rotor to be predicted simultaneously, based on a steady, Reynolds-averaged Navier-Stokes (RANS) solution in the rotating frame of reference. Hence, adjoint sensitivities of these two disciplines can be addressed within a single framework, i.e. the discrete steady adjoint formulization, for this particular case.

\section{A. Review of the Discrete Steady Adjoint Formulization}

The discrete governing flow equations for the steady RANS model can be expressed as

$$
\boldsymbol{R}(\boldsymbol{U}, \boldsymbol{x})=\mathbf{0},
$$

where $\boldsymbol{x}$ is the coordinate vector of volume grid points, $\boldsymbol{U}$ is the vector of flow variables, and $\boldsymbol{R}$ is the vector of flux residuals. Assuming that Eq. (1) is exactly true once a well-converged steady flow solution is obtained, it can be linearized at the flow state $\boldsymbol{U}$ as

$$
\frac{\partial \boldsymbol{R}}{\partial \boldsymbol{U}} \frac{\delta \boldsymbol{U}}{\delta \boldsymbol{x}}=-\frac{\partial \boldsymbol{R}}{\partial \boldsymbol{x}}
$$

where $\delta(\cdot)$ represents an infinitesimal variation.

For any nonlinear, scalar-type objective/constraint function $J(\boldsymbol{U}, \boldsymbol{x})$, which depends on the flow solution and the mesh geometry, its sensitivity w.r.t. a perturbation in the mesh $\boldsymbol{x}$ can be derived by linearization as follows

$$
\frac{\delta J}{\delta \boldsymbol{x}}=\frac{\partial J}{\partial \boldsymbol{U}} \frac{\delta \boldsymbol{U}}{\delta \boldsymbol{x}}+\frac{\partial J}{\partial \boldsymbol{x}}
$$


An alternative way of computing the above sensitivity is the adjoint approach. The vector of adjoint variables, denoted as $\phi$, is defined as the solution of the following adjoint flow equations

$$
\left(\frac{\partial \boldsymbol{R}}{\partial \boldsymbol{U}}\right)^{\top} \boldsymbol{\phi}=\left(\frac{\partial J}{\partial \boldsymbol{U}}\right)^{\top}
$$

where $(\cdot)^{\top}$ is the transpose operation. Then the sensitivity of the function can be expressed using the adjoint flow solution as

$$
\frac{\delta J}{\delta \boldsymbol{x}}=\boldsymbol{\phi}^{\top}\left(-\frac{\partial \boldsymbol{R}}{\partial \boldsymbol{x}}\right)+\frac{\partial J}{\partial \boldsymbol{x}}
$$

Either Eq. (3) or Eq. (5) would yield the mesh sensitivity of the scalar function $J$, which can be regarded as the exact (or analytical) one theoretically. In order to obtain the derivatives of $J$ w.r.t. the shape design parameters to drive a gradient-based optimization, a final step of the matrix multiplication between the mesh sensitivity $\frac{\delta J}{\delta x}$ and the mesh deformation $\frac{\delta x}{\delta \alpha}$ is needed to complete the procedure of the gradient computations. Here, $\alpha$ represents the vector of the shape design parameters. The mesh deformation $\frac{\delta \boldsymbol{x}}{\delta \boldsymbol{\alpha}}$ can be obtained precisely by differentiating the mesh generation code. In this work, however, it is approximated by the second-order central difference. Hence, the final computed derivatives of $J$ w.r.t. $\alpha$ are not the exact linear sensitivities. The nominal order of accuracy of the present adjoint gradient computations is therefore $O\left(h^{2}\right)$, where $h$ is the step size of the shape design parameters.

\section{B. Adjoint Source Term for Shock-Associated Tone Noise}

Predictions of the rotor-alone tone noise based on steady CFD simulations have been demonstrated in previous studies [3, 13]. Modal decomposition of the flow field [14, 15] is often desired to understand the in-duct sound propagation and mode interactions, especially when a lined-duct [3] or a distorted fan inflow [16] is involved. In this study, the ideal scenario, i.e. a fan consisting of identical blades and subjected to a circumferentially uniform inflow, as discussed in Sec. I] for the rotor-alone tone noise generation is considered. The sound intensity expression from [17],

$$
\boldsymbol{I}\left(\boldsymbol{x}_{S}, t\right)=\left(p^{\prime}+\rho_{0} \boldsymbol{u}^{\prime} \cdot \boldsymbol{u}_{0}\right)\left(\boldsymbol{u}^{\prime}+\frac{p^{\prime}}{\gamma p_{0}} \boldsymbol{u}_{0}\right)
$$

is used. Here, $p^{\prime}$ and $\boldsymbol{u}^{\prime}$ are fluctuations of the static pressure and the velocity vector. $\rho_{0}, p_{0}$, and $\boldsymbol{u}_{0}$ are time-averaged values of the flow density, the static pressure, and the velocity vector. $\gamma$ is the ratio of specific heats for an ideal gas. The total power across the surface $S$ can be determined by

$$
W=\frac{1}{T} \int_{T}\left[\iint_{S} \boldsymbol{I}\left(\boldsymbol{x}_{S}, t\right) \cdot \boldsymbol{n} d S\right] d t,
$$

where $\boldsymbol{n}$ is the normal vector of the integral surface, and $T$ is the period for time averaging. The total power calculated 
by Eq. (7) is used as the aeroacoustic objective function for the present fan blade design optimization.

Note that the values of all the variables in Eq. (6) and (7) are "measured" in the stationary frame of reference. In order to build the adjoint source term in Eq. (4) for the total power as the objective, it is necessary to rewrite Eq. (6) and (7) using variables in the rotating frame of reference. Because the unsteadiness observed in the stationary frame of reference is purely a consequence of the rotation of the fan, those fluctuation and mean operations w.r.t. time in Eq. 60 and (7) can be carried out in the rotating frame of reference based on the variation and averaging operations w.r.t. $\theta$ if a cylindrical coordinate system $(x, \theta, r)$ is used. For convenience, an axial cut plane close to the fan inflow boundary is adopted as the integral surface for the total power calculation. The flow data is interpolated onto a polar mesh generated for the axial cut plane. A uniform $\theta$ grid is used to simplify the averaging operation. By using the cylindrical velocity vector, it is straightforward to establish the relation between flow variables in the rotating and stationary frames of reference. Therefore, the time-averaged sound intensity $\overline{\boldsymbol{I}}$ can be rewritten as

$$
\overline{\boldsymbol{I}}(\theta, r) \equiv \frac{1}{T} \int_{T} \boldsymbol{I}\left(\boldsymbol{x}_{S}, t\right) d t=\frac{1}{2 \pi} \int_{0}^{2 \pi}\left[p^{\prime}+\rho_{0} \boldsymbol{u}^{\prime} \cdot\left(\boldsymbol{u}_{0}+\boldsymbol{V}_{\Omega}\right)\right]\left[\boldsymbol{u}^{\prime}+\frac{p^{\prime}}{\gamma p_{0}}\left(\boldsymbol{u}_{0}+\boldsymbol{V}_{\Omega}\right)\right] d \theta
$$

where $V_{\Omega}=(0, \Omega r, 0)^{\top}$ and $\Omega$ is the shaft rotating speed. All variables on the right-hand-side of Eq. (8) are in the rotating frame of reference, and $(\cdot)^{\prime}$ and $(\cdot)_{0}$ operators are now defined for $\theta$. The total power, by integrating $\overline{\boldsymbol{I}}$ over the axial cut plane, is

$$
W=2 \pi \int_{r_{\min }}^{r_{\max }} \overline{\boldsymbol{I}}(r) \cdot \boldsymbol{n} d r
$$

Going backwards from flow data on the axial cut plane to that on the original CFD grid would depend on the interpolation method used in practice. In general, it can be expressed, for any variable $f$, as matrix multiplication,

$$
\boldsymbol{f}_{\text {axial cut }}=\boldsymbol{A}_{N \times M} \boldsymbol{f}_{\mathrm{CFD} \text { grid }}
$$

where $\boldsymbol{A}_{N \times M}$ is the weighting matrix for interpolation, $N$ is the number of grid points on the axial cut plane, and $M$ is the number of grid points for the original CFD mesh.

The adjoint source term for the total power can be formally derived based on Eq. (8)- (10). The result is summarized as follows, 


$$
\begin{aligned}
& \frac{\partial W}{\partial \boldsymbol{U}} \equiv\left(\frac{\partial}{\partial \rho}, \frac{\partial}{\partial u_{x}}, \frac{\partial}{\partial u_{\theta}}, \frac{\partial}{\partial u_{r}}, \frac{\partial}{\partial p}, \frac{\partial}{\partial \tilde{v}}\right)^{\top} W \\
& =2 \pi \int_{r_{\min }}^{r_{\max }}\left[\begin{array}{c}
\frac{\partial \overline{\boldsymbol{I}}}{\partial \rho_{0}} \frac{d \rho_{0}}{d \rho} \\
\frac{\partial \overline{\boldsymbol{I}}}{\partial u_{x 0}} \frac{d u_{x 0}}{d u_{x}}+\frac{\partial \overline{\boldsymbol{I}}}{\partial u_{x}^{\prime}} \frac{d u_{x}^{\prime}}{d u_{x}} \\
\frac{\partial \overline{\boldsymbol{I}}}{\partial u_{\theta 0}} \frac{d u_{\theta 0}}{d u_{\theta}}+\frac{\partial \overline{\boldsymbol{I}}}{\partial u_{\theta}^{\prime}} \frac{d u_{\theta}^{\prime}}{d u_{\theta}} \\
\frac{\partial \overline{\boldsymbol{I}}}{\partial u_{r 0}} \frac{d u_{r 0}}{d u_{r}}+\frac{\partial \overline{\boldsymbol{I}}}{\partial u_{r}^{\prime}} \frac{d u_{r}^{\prime}}{d u_{r}} \\
\frac{\partial \overline{\boldsymbol{I}}}{\partial p_{0}} \frac{d p_{0}}{d p}+\frac{\partial \overline{\boldsymbol{I}}}{\partial p^{\prime}} \frac{d p^{\prime}}{d p} \\
0
\end{array}\right] \cdot \boldsymbol{n r} d r \boldsymbol{A}_{N \times M} .
\end{aligned}
$$

It is assumed here that the Spalart-Allmaras (SA) turbulence model is used, and the gradient component for the SA variable $\tilde{v}$ is preserved for completeness. For any variable, $\frac{d(\cdot)^{\prime}}{d(\cdot)}=\boldsymbol{E}_{N}-\frac{d(\cdot)_{0}}{d(\cdot)}$, where $\boldsymbol{E}_{N}$ is the identity matrix of size $N$. In addition, velocity transformation between the cylindrical and Cartesian components may be needed if the CFD solver uses a Cartesian coordinate system.

\section{Numerical Solver}

The Rolls-Royce HYDRA-CFD code [18] is used as the numerical tool in the present study. It is a coupled suite of non-linear, linear, and adjoint solvers for hybrid unstructured meshes. The spatial discretization uses a MUSCL-based flux-differencing algorithm based on an efficient edge-based data structure. For steady non-linear problems, the discrete flow equations are iterated towards a satisfactory residual level using either an explicit or an implicit Runge-Kutta pseudo-time-marching scheme. The convergence to the steady state can be accelerated using an element-collapsing multigrid algorithm. The linear and adjoint flow equations are solved using the same algorithms for spatial discretization and pseudo-time-marching. The generalized minimal residual method can be used to stabilize the linear and adjoint solvers for those cases where instabilities arise in the iteration. All the HYDRA solvers are parallelized based on domain decomposition.

The current HYDRA version supports many objective options for the adjoint sensitivity analysis, which are mainly aerodynamic and thermodynamic performance parameters. This paper develops the first adjoint simulation for an aeroacoustic objective that can be used for the transonic fan design optimizations in turbomachinery and therefore enriches the multidisciplinary capabilities of the adjoint solver.

\section{Mesh Study and Simulation Validation}




\section{A. Case Description}

The case under the present investigation is a transonic research fan designed by Rolls-Royce. It is a low-speed, high bypass-ratio, scaled-down fan model with 18 rotor blades for rig testing purpose, and the blade is thicker than a conventional fan blade meant to represent an engine size composite fan blade.

The computational domain and CFD boundary conditions used for the steady RANS simulations of the fan are shown in Fig. 1. A downstream splitter is included in the fan rotor-alone configuration, which enables the bypass ratio to be considered. Non-reflecting subsonic inflow and outflow boundary conditions based on the method of characteristics are imposed on the fan inlet, the bypass exit, and the engine core exit, respectively. At the inlet, radial profiles of total pressure, total temperature, and inflow angles are specified. At the exits, two fixed exit capacity (i.e. exit flow function) values are given, together with radial equilibrium applied on each exit boundary. This is believed to be a reasonable simulation of the operating condition of a blade row in realistic, multistage compression configurations [19], and can roughly ensure that the optimization is performed on a working line of the original fan. The one-equation SA turbulence model is used. The fan speed and the inflow and outflow boundary conditions used in the present study are corresponding to the aerodynamic design point of the fan, which lies on the cruise working line.

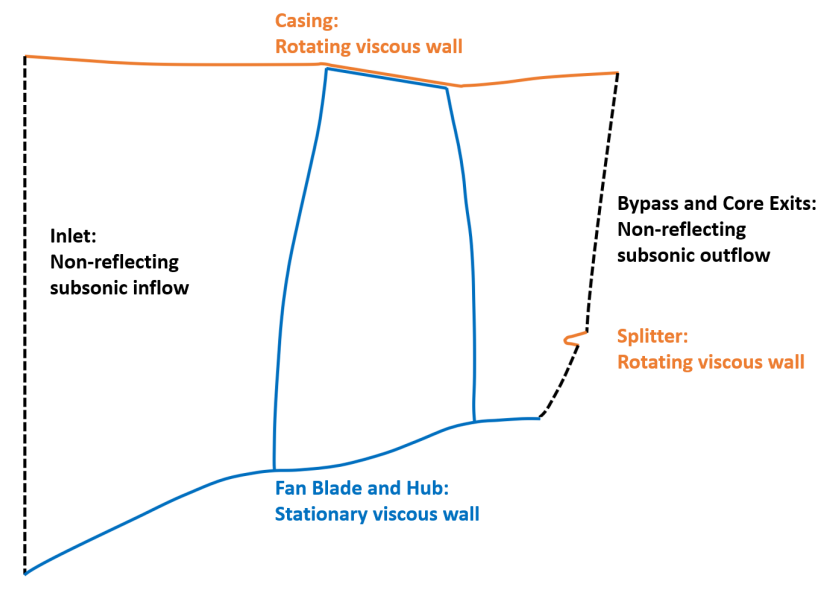

Fig. 1 Computational domain and boundary conditions.

The geometric parameterization method used for the present study is the engineering parameters implemented in the Roll-Royce PADRAM code [20]. Seven parameters, including axial sweep (XCEN, mm), circumferential lean $\left(\mathrm{DELT}^{\circ}{ }^{\circ}\right.$ ), solid body rotation $\left(\mathrm{SKEW},{ }^{\circ}\right)$, leading-edge re-cambering $\left(\mathrm{LEMO},{ }^{\circ}\right)$, trailing-edge re-cambering $(\mathrm{TEMO}$, ${ }^{\circ}$ ), the chord position for LEMO blending (LEBP, dimensionless), and the chord position for TEMO blending (TEBP, dimensionless), are used to perturb the 2D sectional blade profile. Note that either of the last two blending parameters is active only when the corresponding LEMO or TEMO is nonzero. The seven parameters are specified at five radial positions $(25 \%, 50 \%, 75 \%, 87.5 \%$, and $100 \%$ blade span) and then interpolated through the whole span onto each blade section using the piecewise cubic spline. This gives a design space of 35 design variables. 


\section{B. Mesh Dependence}

PADRAM [20] is used for the multiblock structured mesh generation. An H-O-H mesh topology is applied to the blade-to-blade section, and then a user-defined distribution is used for the radial stacking. Uniform grids are applied both axially and circumferentially to the rotor upstream H (USH) block to reduce numerical dissipation for the noise calculation. The radial mesh is clustered towards two endwalls to resolve the hub and casing wall boundary layers. The O-mesh around the fan blade is designed to have near-wall $y^{+}$values of the order of one. Adequate mesh cells are added in the blade tip gap region to capture the tip leakage flow.

It is necessary to conduct a mesh dependence study before doing optimization. Special attention has been paid to the effects of the axial grid number of the USH block and the radial grid number on the predictions of both aerodynamic and aeroacoustic performance parameters, while the circumferential grids were kept the same. The grids that have been tested are marked with " $\times$ ” in Tab. 1. For example, mesh A3R2 stands for a mesh that has 92 axial points in the USH block and 130 radial points.

Table 1 Grids tested for mesh dependence

\begin{tabular}{|cc|c|c|c|c|c|}
\hline \multicolumn{2}{|r|}{$\begin{array}{r}\text { Axial grids } \\
\text { in USH }\end{array}$} & A1 & A2 & A3 & A4 & A5 \\
Radial grids & 31 & 61 & 92 & 122 & 153 \\
\hline R1 & 110 & & $\times$ & & $\times$ & \\
\hline R2 & 130 & & & $\times$ & $\times$ & \\
\hline R3 & 150 & $\times$ & $\times$ & $\times$ & $\times$ & $\times$ \\
\hline R4 & 160 & & & & $\times$ & \\
\hline \hline \multicolumn{7}{r|}{ Number of circumferential grids in USH: 106} \\
\hline
\end{tabular}
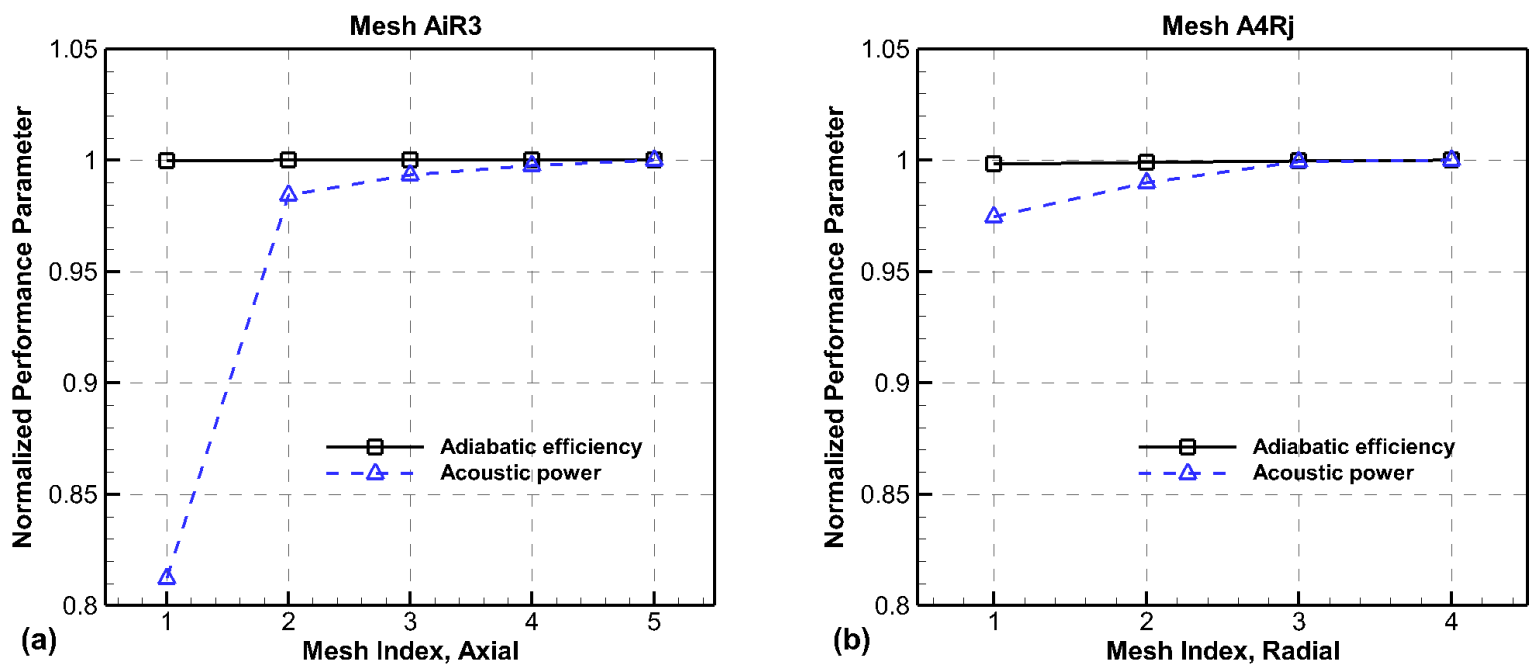

Fig. 2 (a) Axial and (b) radial grid dependence for the predictions of performance parameters. 
Figure 2 shows the mesh dependence results for the normalized adiabatic efficiency and acoustic power predicted by using mesh $\mathrm{A} i \mathrm{R} 3$ and $\mathrm{A} 4 \mathrm{R} j$, where $i=1,2,3,4,5$, and $j=1,2,3,4$. It shows that satisfactory, mesh-independent predictions (less than $0.25 \%$ changes relative to the finest mesh predictions) of the aerodynamic and aeroacoustic parameters can be obtained by using mesh A4R3, which consists of 6.013 million points for the present single-bladepassage configuration. The USH block of mesh A4R3 has an estimated resolution of 17 points per wavelength for 3BPF tone. Figure 2 also highlights that the aeroacoustic performance prediction is more sensitive to the axial grid density of the USH block as well as the radial grid density, compared with the prediction of aerodynamic performance.
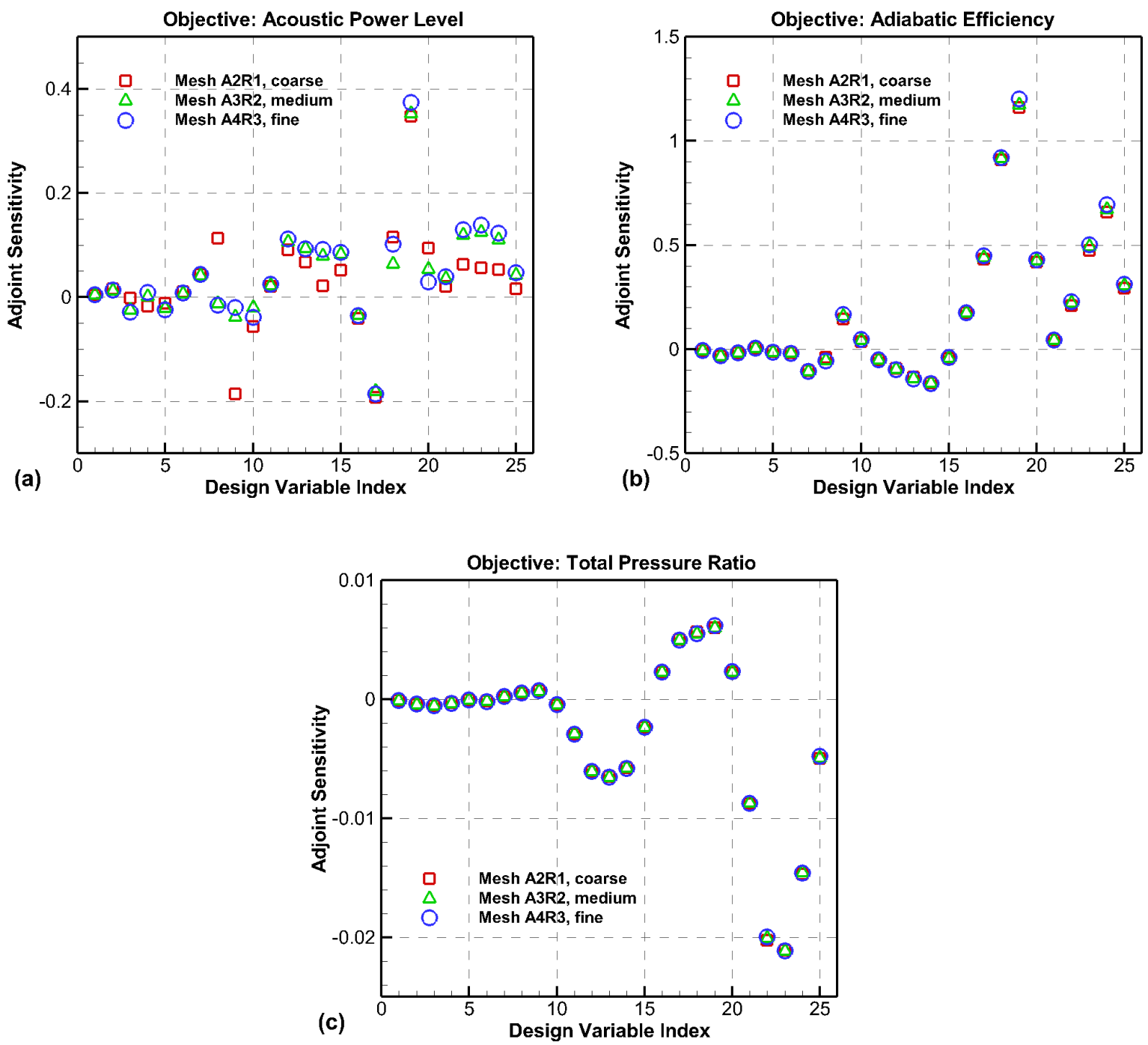

Fig. 3 Mesh dependence for adjoint sensitivities of (a) acoustic power level, (b) adiabatic efficiency, and (c) total pressure ratio.

For the adjoint-based optimization, it is more important to ensure the calculated adjoint gradients to be meshindependent as far as possible. Three meshes, i.e. A2R1, A3R2, and A4R3, are then tested for adjoint simulations 
regarding the fan inlet total acoustic power level, adiabatic efficiency, and total pressure ratio. Here, the adjoint simulations are carried out for the datum fan blade and therefore LEBP and TEBP are inactive. The calculated gradients are compared in Fig. 3. The aeroacoustic adjoint gradients are also seen to be more sensitive to the mesh density than the adjoint gradients for aerodynamic objectives. Nevertheless, Fig. 3 suggests that mesh A4R3 can be regarded as a proper mesh for the present adjoint-based optimization since it can provide almost converged adjoint gradients for both aeroacoustic and aerodynamic objectives.

\section{Simulation Validation}

The fan CFD simulation is validated by a comparison of spanwise profiles of the fan aerodynamic performance between the present prediction and experimental data [21]. Figure 4 shows that the total pressure ratio spanwise distribution agrees well with the experimental result, while the fan efficiency is under-predicted (within $2 \%$ ) over the mid-span range. The rapid drop of the efficiency above $60 \%$ of the blade span is mainly caused by the shock-induced loss mechanisms [22], where not only direct entropy production occurs across the shock but also viscous losses are generated as a result of the shock-boundary layer interactions.
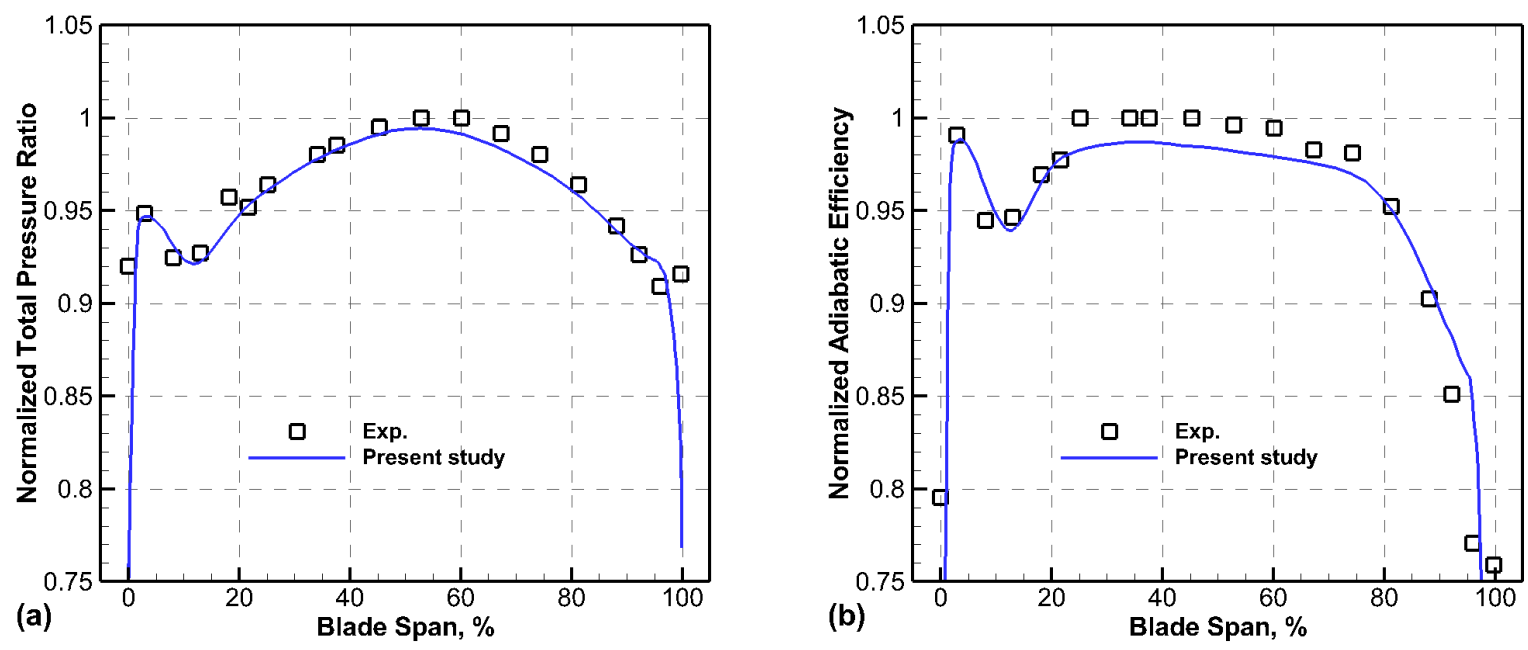

Fig. 4 Comparisons of the spanwise performance profiles of (a) total pressure ratio and (b) adiabatic efficiency.

The calculated adjoint gradients are compared against their counterparts directly obtained by second-order finite difference (FD) approximation. Figure 5 shows the comparison results for two meshes, i.e. A2R1 and A4R3, for the datum blade shape. Besides, in order to validate the adjoint sensitives to the 10 design variables corresponding to LEBP and TEBP, another comparison is made based on an arbitrarily perturbed blade using mesh A4R3 and the results are shown in Fig. 6 Overall, reasonable agreements between the adjoint gradients and the FD approximations based on the same meshes are achieved for each objective. This validates the aeroacoustic and aerodynamic adjoint simulations in 
the present study. It is noted that, for all the cases tested here, the adjoint and the FD approximated sensitivities to four TEMO parameters (design variable 21 to 24) and two TEBP parameters (design variable 32 and 33) have distinct deviations in magnitudes yet the same sign. Nevertheless, it is believed that the adjoint simulations developed in this paper can efficiently provide the Jacobians to determine correct searching directions for the optimization.
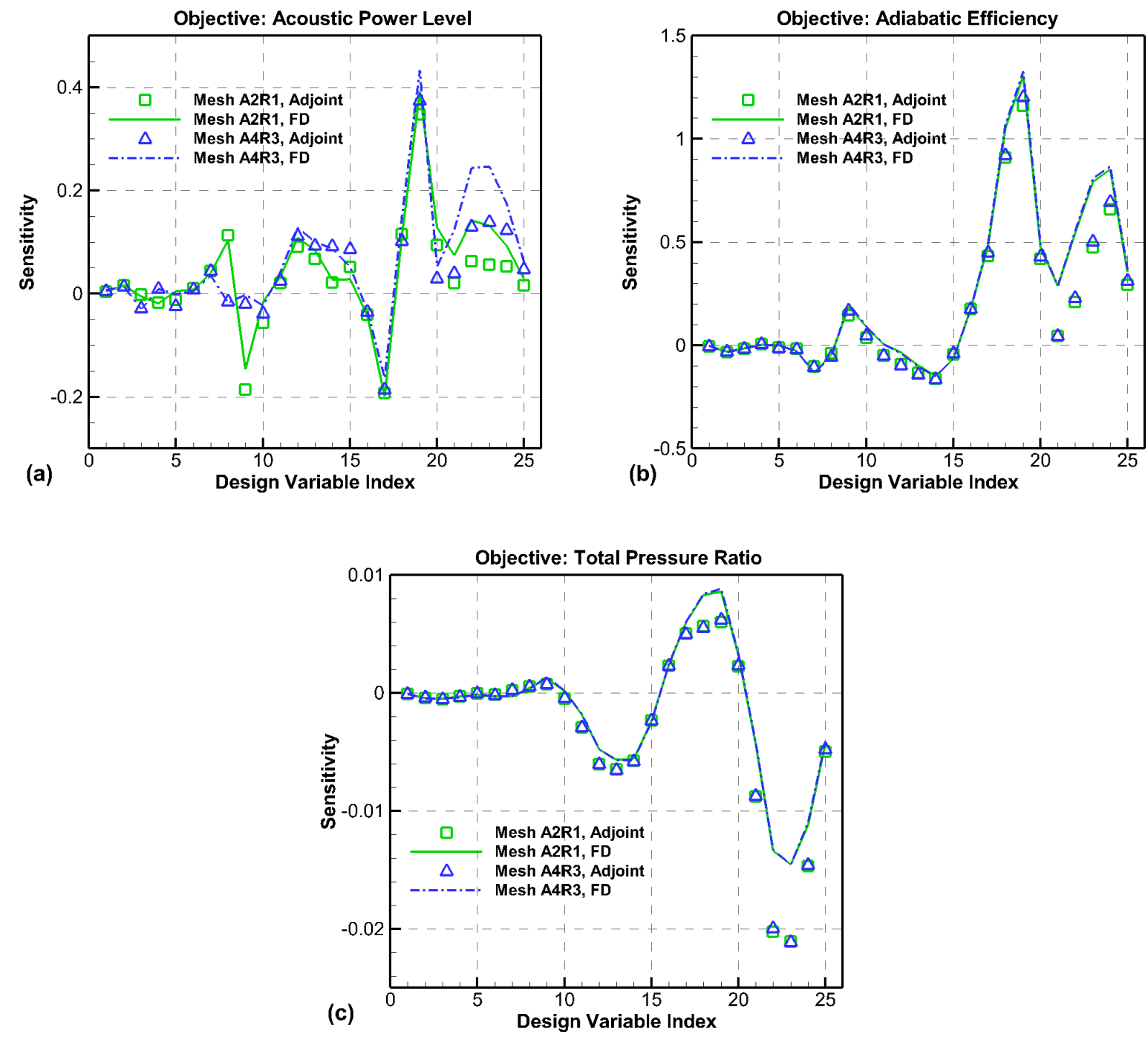

Fig. 5 Comparisons of the adjoint and the FD sensitivities of (a) acoustic power level, (b) adiabatic efficiency, and (c) total pressure ratio for the datum fan blade.

\section{Adjoint-Based Design Optimization}

\section{A. Optimization Strategy}

The aeroacoustic and aerodynamic optimization of the fan blade is posed as an MOO problem, and the two objectives considered are the fan inlet acoustic power level $P W L\left(\mathrm{~dB}\right.$ ref. $10^{-12}$ Watts) and the adiabatic efficiency $\eta$. The solution 

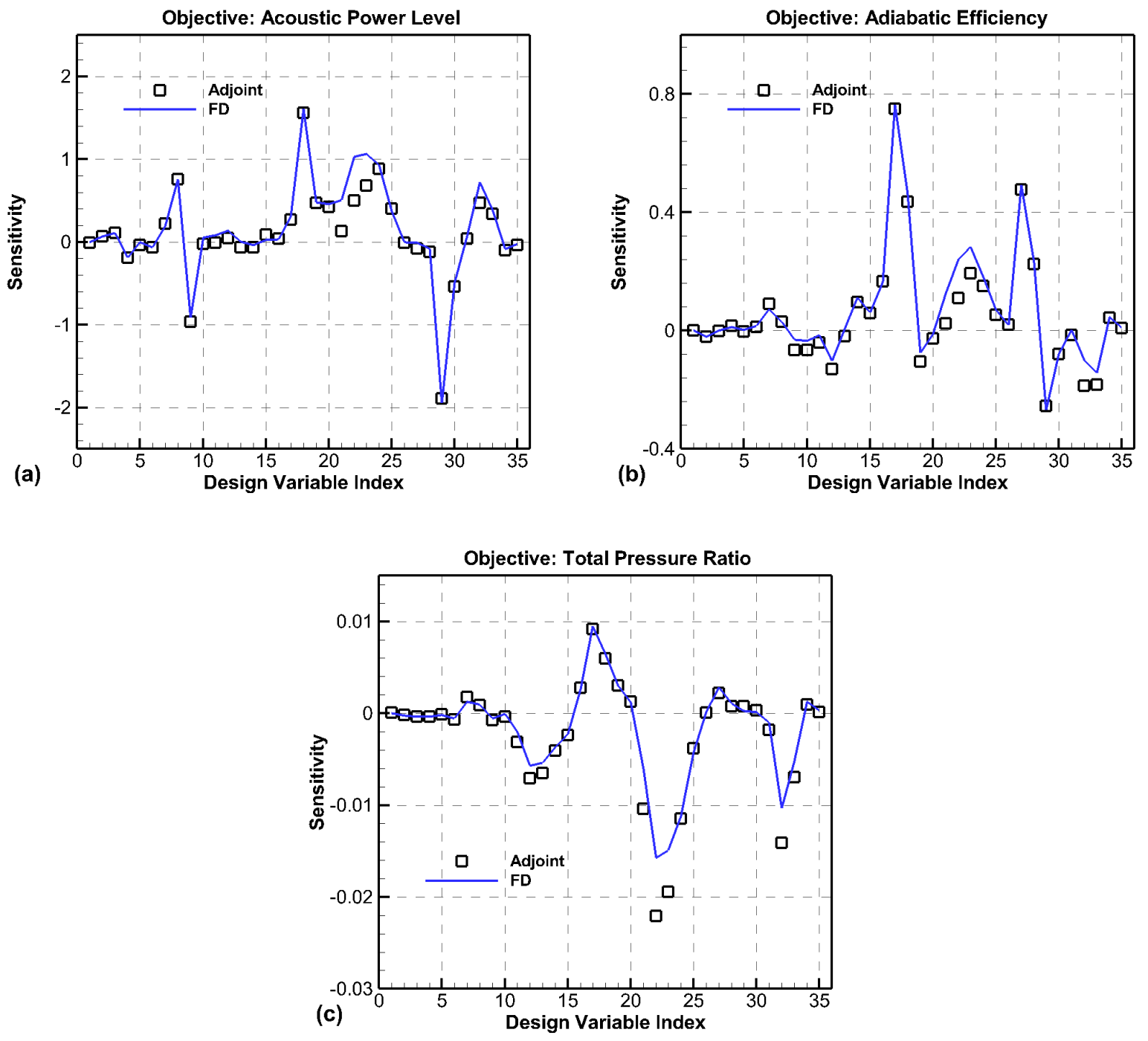

Fig. 6 Comparisons of the adjoint and the FD sensitivities of (a) acoustic power level, (b) adiabatic efficiency, and (c) total pressure ratio for an arbitrarily perturbed blade.

of an MOO problem is the so-called Pareto front that represents the tradeoffs between different objectives. In practice, there are two strategies to generate the Pareto front. One is to tackle the MOO problem directly by using heuristic algorithms, such as the multi-objective genetic algorithms [23, 24]. The advantages are that a list of nondominated solutions is evolved during the iterations, and the algorithm can be tuned to prevent the optimization from being trapped in local optima. However, due to their population-based nature, it is computationally expensive to apply heuristic algorithms with high-fidelity CFD simulations for large-scale optimization problems in turbomachinery. The other strategy is to reduce the MOO problem to a parameterized scalar optimization (SO) problem by various scalarization techniques [25-27] so that the SO problem can be solved consecutively over the set of the scalarization parameters to depict the Pareto front in a point-by-point manner. 
The weighted sum method is used extensively for MOO problem not only to generate a representation of the Pareto front but also to provide a single Pareto optimal solution with a priori articulation of preferences [25]. In the present study, the linear weighted sum method is employed to define a composite objective function of $P W L$ and $\eta$,

$$
F(\boldsymbol{d} ; \alpha)=\alpha g(\boldsymbol{d})+(1-\alpha) h(\boldsymbol{d})
$$

where $g$ and $h$ are rescaled functions corresponding to $P W L$ and $1-\eta, \boldsymbol{d}$ is the vector of the 35 design variables, and $\alpha$ is the weighting factor. Theoretically, the complete Pareto front is guaranteed using the linear weighted sum method if the MOO problem is convex [25]. Since it is impossible to know the convexity a priori, minimizing the composite function $F$ over a sample of $\alpha$ in $[0,1]$ might only produce a sample of the noise-efficiency Pareto front. Each design variable in $\boldsymbol{d}$ is bounded in its range. The optimization algorithm used in the present paper is the sequential least squares programming (SLSQP) [28], which can effectively handle linear and nonlinear, equality and inequality constraints. A Boolean constraint is applied to the optimizations throughout this work to rule out those design candidates of either meshing failure or poor CFD convergence.

The optimization process is automated using Python scripting, and the workflow is shown in Fig. 7

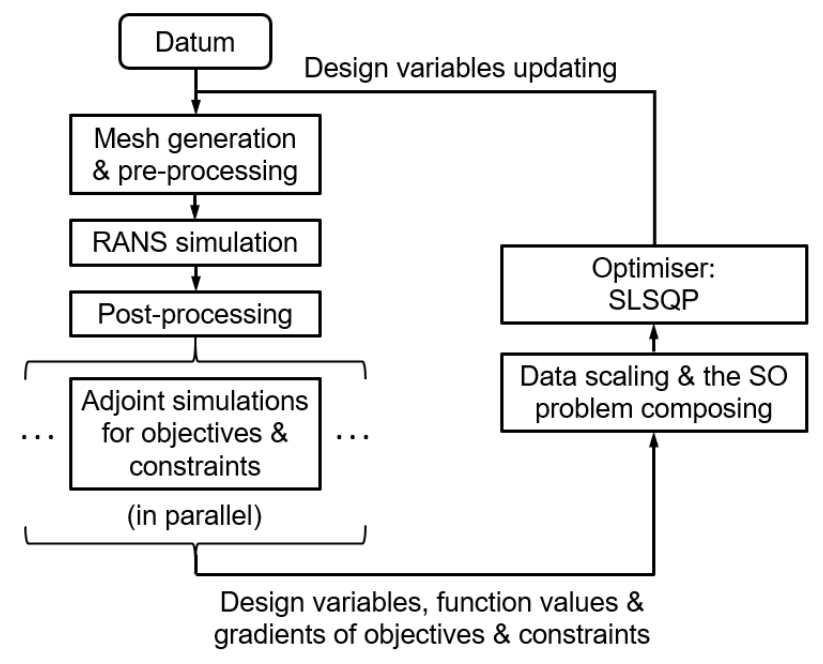

Fig. 7 Optimization workflow.

\section{B. Single-Objective Aeroacoustic Optimization}

First, an unconstrained, aeroacoustic optimization is performed aiming at reducing the $P W L$ of the shock-associated BPF tones. Figure 8 shows the convergence history of the optimization. It has demonstrated how the aeroacoustic adjoint simulation developed in the present study can facilitate the low-noise fan blade design. A significant $P W L$ reduction of around $14 \mathrm{~dB}$ is achieved, accompanied by a $1.9 \%$ efficiency loss and a $2.7 \%$ increase in the total pressure ratio. The mass flow is also increased as a result of moving the fan operation point along the working line. Two nosedives of the 
$P W L$, after the first iteration and the seventh iteration, can be seen from the optimization history.
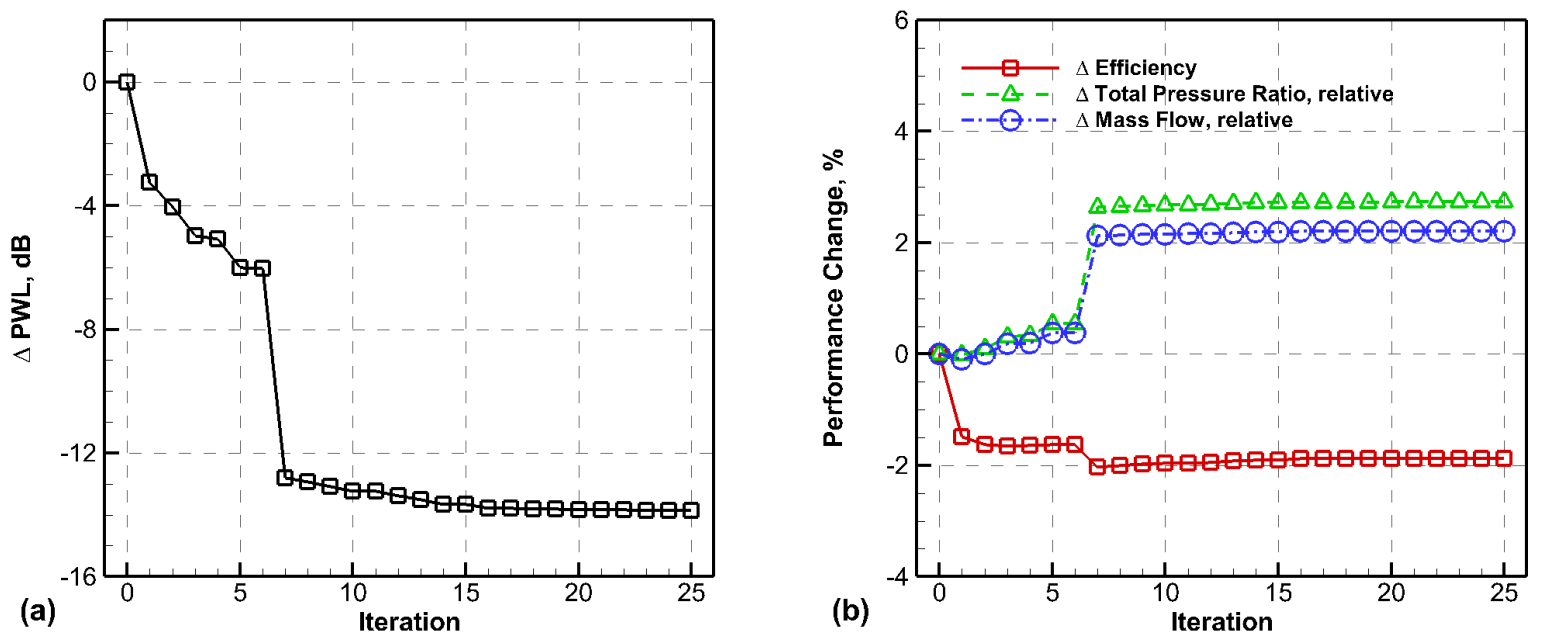

Fig. 8 Unconstrained single-objective aeroacoustic optimization history curves of (a) the $P W L$ and (b) the aerodynamic performance parameters.

The fan blade geometries at iteration 1, 6, 7, and 25 are compared with the datum in Fig. 9 The most significant shape change responsible for the shock-associated noise reduction is believed to be the compound sweep profile of the blade leading edge obtained by the optimization, which directly determines the cross-sectional distribution of the initial shock close to the rotor. The blade leading edge is seen to consist of a forward-swept portion below $75 \%$ span, a rearward-swept region between $75 \%$ and $87.5 \%$ span, and finally, a slightly rearward-swept tip region between $87.5 \%$ and $100 \%$ span. It is interesting to note the strong similarities between the present compound leading-edge sweep pattern, the compound sweep blade resulting from a "shockless" leading edge concept proposed by Bliss et al. [29], and the low-shock tone noise blade designed by Wilson et al. [3]. In fact, the compound leading-edge sweep has the ability to "manipulate" the shock distribution near the rotor face in a specific way so that the acoustic energy is redistributed between the duct modes [3]. This will be discussed in detail in Sec. IV.E The nosedive of the $P W L$ after the first iteration is caused by the switch-on of the leading-edge sweep pattern.

Other noteworthy shape changes are the lean towards the rotating direction at 75\% span, the increased camber of the blade trailing edge between $25 \%$ and $87.5 \%$ span, and the decreased stagger angles (between the chord line and the engine axis) between $25 \%$ and $75 \%$ span. These features apparently have the overall effect of opening up the blade passage, and therefore the mass flow is anticipated to increase. Due to the working line constraint imposed by the use of fixed capacities on the outflow boundaries, the total pressure ratio would increase as well. It might also be explained as a result of probable excessive flow turning and increased angles of attack over a large portion of the blade span, which in turn would increase the work done on the fluid. Meanwhile, increasing the mass flow implies increasing the axial 


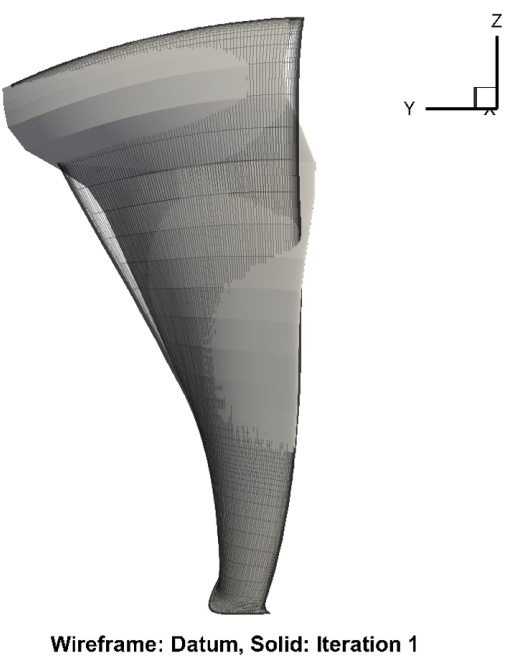

(a)

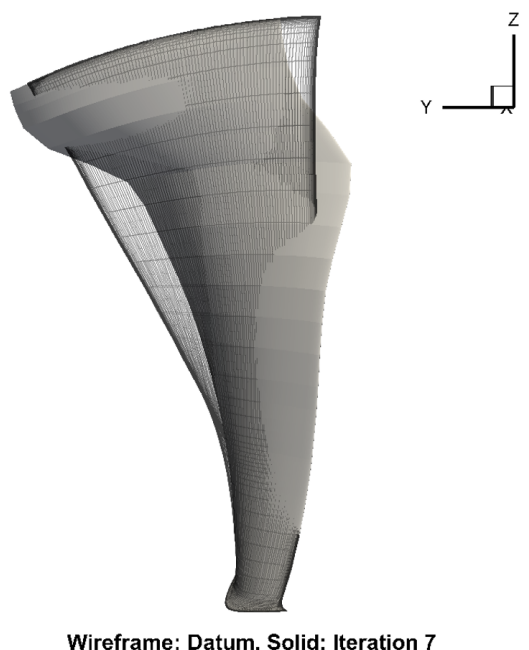

(c)

Wireframe: Datum, Solid: Iteration 7

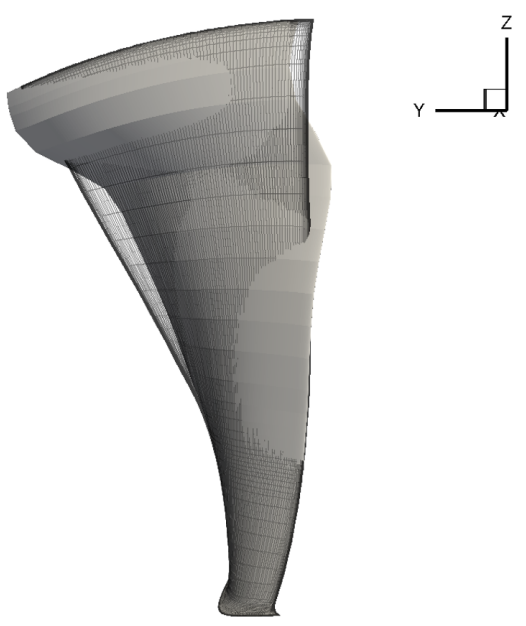

Wireframe: Datum, Solid: Iteration 6

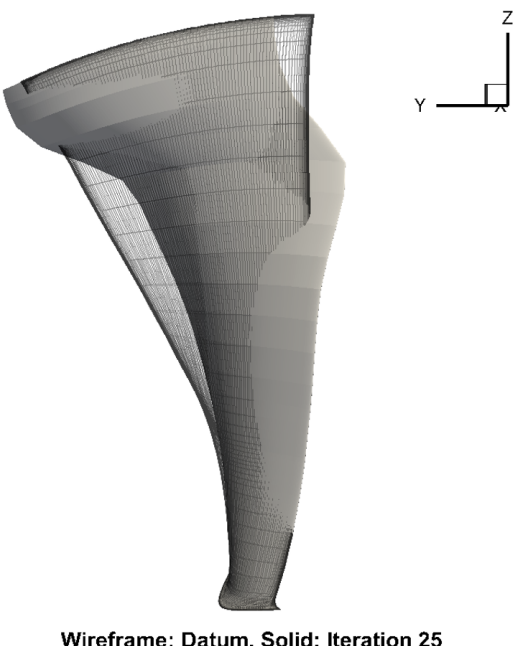

(d)

Wireframe: Datum, Solid: Iteration 25

\section{Fig. 9 Blade shape comparisons between the datum and the geometries obtained at iteration 1, 6, 7, and 25 .}

and the relative Mach numbers of the approach flow, and this change of the velocity triangle also makes the helical shock front turning towards the rotor face. It can then be inferred from two-dimensional shock propagation models [5, 30, 31] that, the so-called "time of flight" of the shock waves over a fixed axial distance would increase and so would the nonlinear attenuation.

To summarize, it seems that the unconstrained aeroacoustic optimization had exploited two mechanisms for the shock tone noise reduction, i.e. the manipulation of the initial shock generation, and the enhancement of the nonlinear attenuation during the propagation process. The $P W L$ nosedive, as well as the mass flow soar, from iteration 6 to iteration 7 is seen to be caused by sudden increases in the amplitudes of the lead-edge sweep and in the blade passage area driven by the optimizer, which confirms the combined effect of the two mechanisms. 
In practice, design optimization of a fan blade should be conducted at specified operating points on the fan map, and this implies that the mass flow and the total pressure ratio at the design point are seen as design requirements. In the present study, the use of fixed capacities on the outflow boundaries is equivalent to imposing a constraint condition on the mass flow and the total pressure ratio, which approximatively represents the fan cruise working line. Therefore, a restriction on either the mass flow or the total pressure ratio could make the final design satisfying the specified operating conditions. Here, a tight constraint is imposed on the total pressure ratio $P R$,

$$
\left(\frac{P R-P R_{\text {datum }}}{P R_{\text {datum }}}\right)^{2} \leq(0.25 \%)^{2} .
$$

Two strategies are used to meet the requirement of Eq. [13]. One is to re-conduct an adjoint-based, aeroacoustic optimization in the same design space initialized from the baseline geometry while with the $P R$-constraint switched on. As shown in Fig. 10 , the $P R$-constrained, noise optimization stopped after 11 iterations, and only achieved a $5.5 \mathrm{~dB}$ $P W L$ reduction. The fan operating point was well-controlled within the tolerance during the whole optimization process which, however, might not be necessary. Nevertheless, the shape comparison between the constrained optimized blade and the unconstrained optimized ones in Fig. 11 might confirm the compound leading-edge sweep pattern to be a low shock tone noise configuration. The constrained optimized blade has smaller amplitudes of the lead-edge sweep, a smaller blade passage area, and therefore a lesser noise reduction.
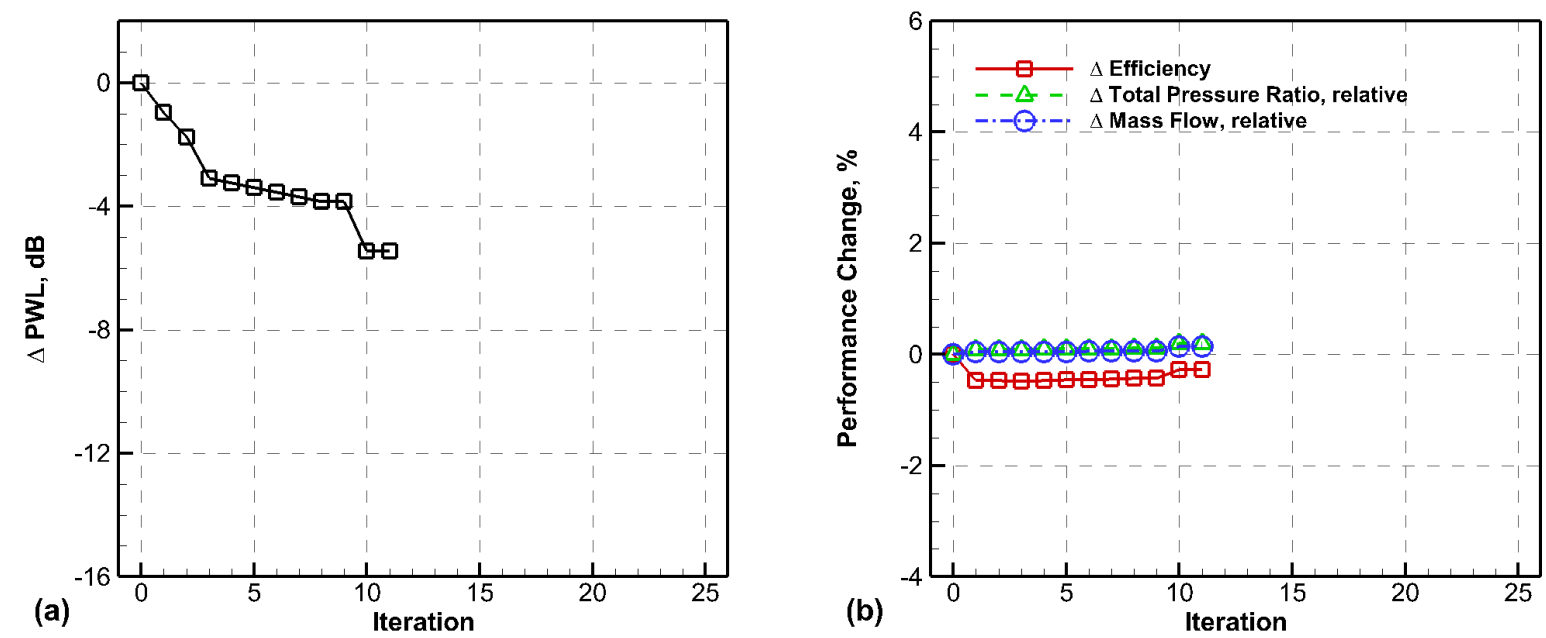

Fig. 10 Constrained single-objective aeroacoustic optimization history curves of (a) the $P W L$ and (b) the aerodynamic performance parameters.

The other is a $P R$-recovery strategy based on the previous unconstrained aeroacoustic optimization result. The idea is to bring the operating point of the unconstrained optimized blade back to that of the datum blade by solving a $P R$ "minimization" problem. 


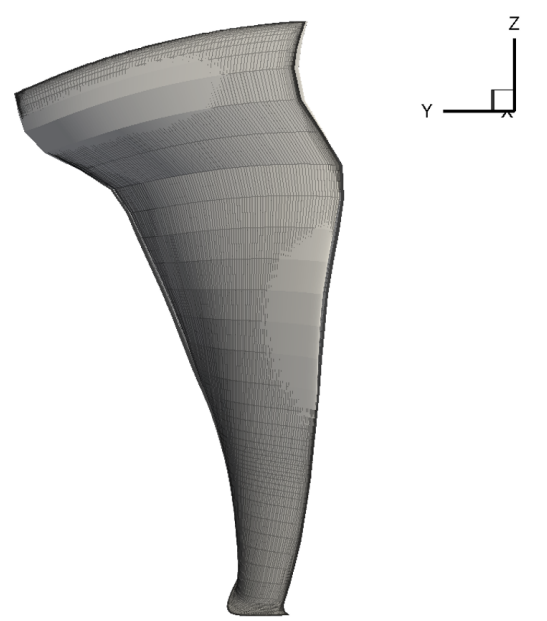

(a) Wireframe: Constrained, Solid: Unconstrained - Iteration 6

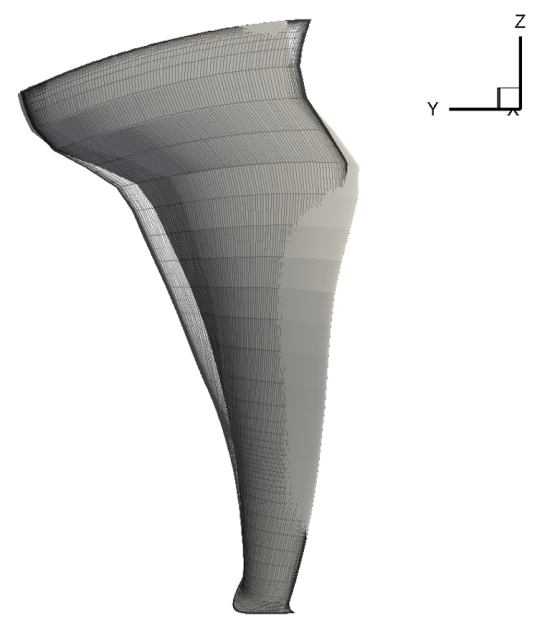

Wireframe: Constrained, Solid: Unconstrained - Iteration 25

Fig. 11 Blade shape comparison between the constrained and unconstrained optimization.
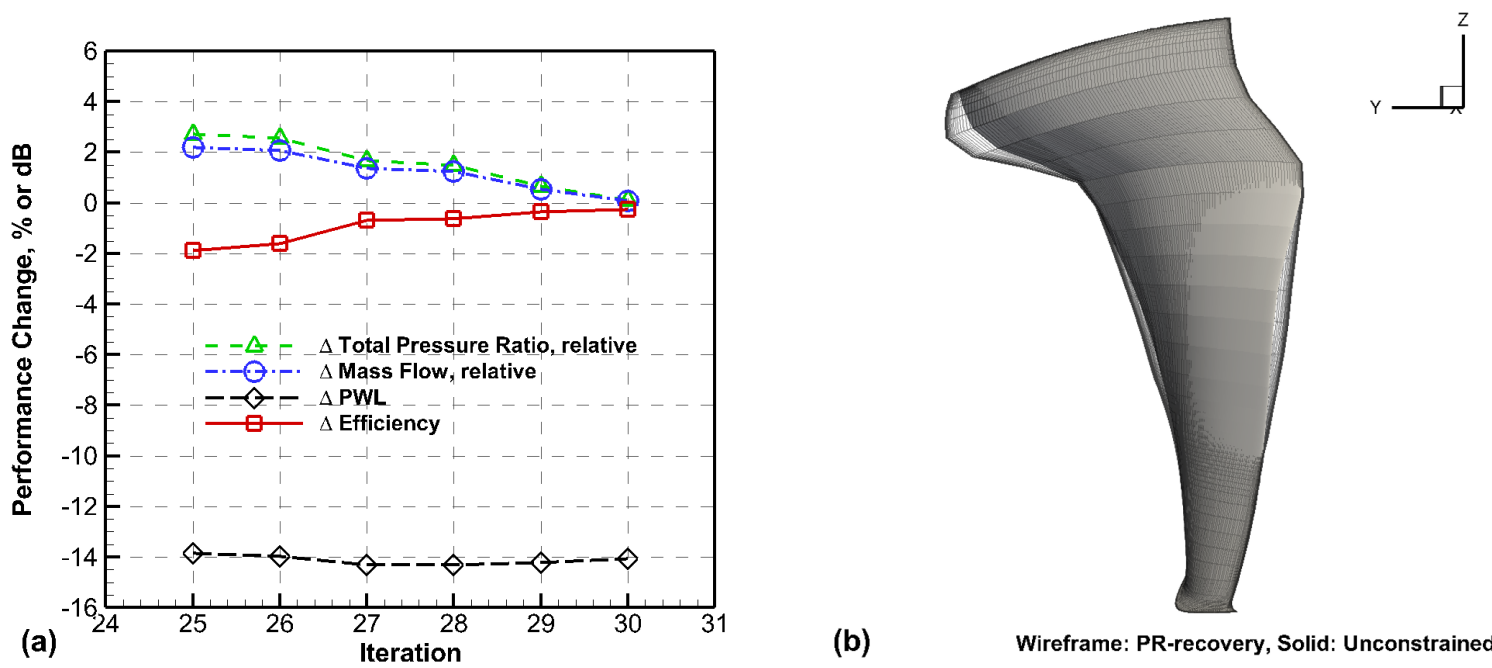

(b)

Wireframe: PR-recovery, Solid: Unconstrained

Fig. 12 The $P R$-recovery optimization result: (a) history curves of the performance parameters, and (b) blade shape comparison before and after the $P R$-recovery optimization.

$$
\begin{array}{cl}
\min _{\boldsymbol{d}_{\text {sub }}} & \left(\frac{P R-P R_{\text {datum }}}{P R_{\text {datum }}}\right)^{2} \\
\text { s.t. } & P W L \leq P W L_{\text {iter25 }} \\
& \boldsymbol{d}_{\text {sub }} \in \mathbb{D}_{\text {sub }}
\end{array}
$$

Here, $P W L_{\text {iter25 }}$ is the sound power level of the unconstrained optimized blade from which the optimization problem 
Eq. (14) is started. A sub-design space of 7 design variables, $\mathbb{D}_{\text {sub }}$, is used for the $P R$-recovery optimization, which consists of the SKEW parameters at 25\% and 50\% span, the LEMO parameters at 25\% and 50\% span, and the TEMO parameters at $25 \%, 87.5 \%$, and $100 \%$ span. These design parameters are selected because they could have immediate impacts on the control of the passage mass flow and the work done on the fluid. Figure 12 shows the history of the $P R$-recovery optimization and the final blade shape. Note that there is no need for the optimization to fully converge as long as Eq. (13) is satisfied. The final blade successfully restored the operating point to that of the original fan, and meanwhile, it achieved nearly the same noise reduction $(14.1 \mathrm{~dB})$ yet with a reduced efficiency penalty $(0.25 \%)$.

A remark on the optimality of the unconstrained and constrained aeroacoustic optimization results could be useful for a better glimpse of the present low-noise blade design problem. Figure 13 shows the history curves of the gradient norms of the objective $P W L$ for the unconstrained and constrained optimizations. The overall increase of the $P W L$ gradient norms for the two cases might suggest that the present optimizations were not in a convex region of the noise objective function. Moreover, intuitively it can be expected that further increases in the blade leading-edge sweep would likely lead to more reductions in the shock-associated noise [29]. The reason why the two optimizations stopped, therefore, was not because of approaching a local minimum but because the line search at the last iterations cannot reduce the objective any further. The failure of the final line search reflected possible limitations of the present optimizations, such as insufficient accuracy of the adjoint gradient and the use of unified mesh settings in the optimization process.

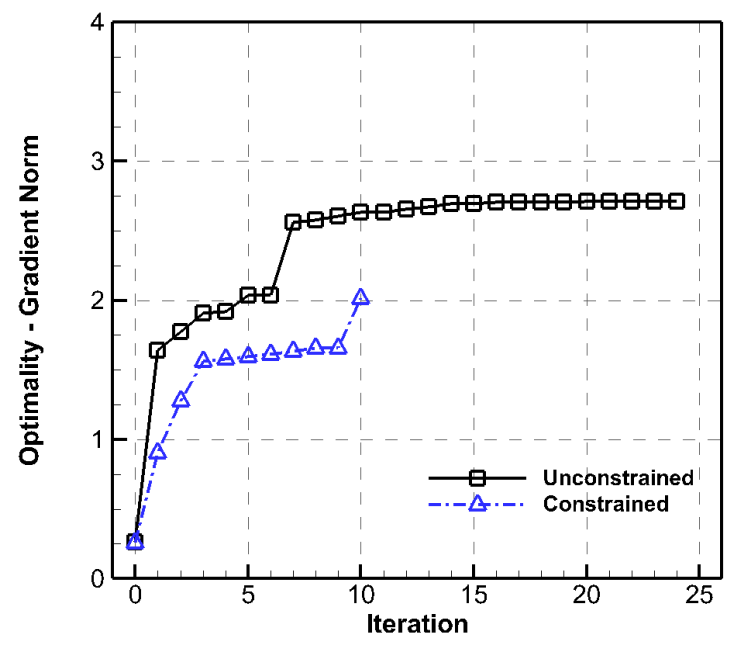

Fig. 13 History curves of the gradient norms of the objective $P W L$ for the unconstrained and constrained aeroacoustic optimizations.

\section{Single-Objective Aerodynamic Optimization}

The composite function $F$ is then minimized with and without the $P R$ constraint for the other limiting case when $\alpha=0$, i.e. the single-objective aerodynamic optimization to increase the fan efficiency. The optimization histories are 
shown in Fig. 14, and the final blade shapes are compared in Fig. 15. The unconstrained and constrained optimizations result in a $0.8 \%$ and a $0.76 \%$ increase in the efficiency, respectively, which are both seen as appreciable benefits considering the baseline efficiency of the Rolls-Royce research fan used in the present study is quite high. The specified fan operating condition is satisfied within the tolerance for the two efficiency-optimized designs. Besides, they both show slight noise reductions.
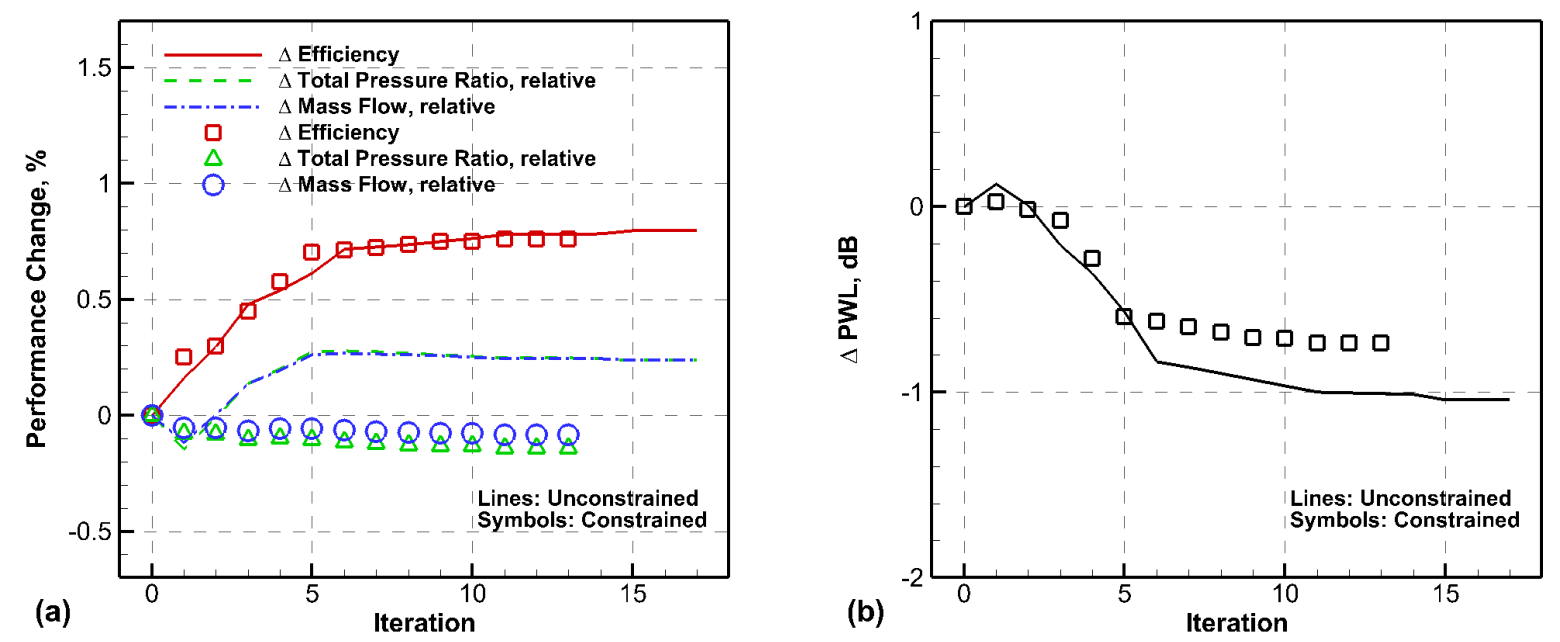

Fig. 14 Single-objective aerodynamic optimization history curves of (a) the aerodynamic performance parameters and (b) the $P W L$.
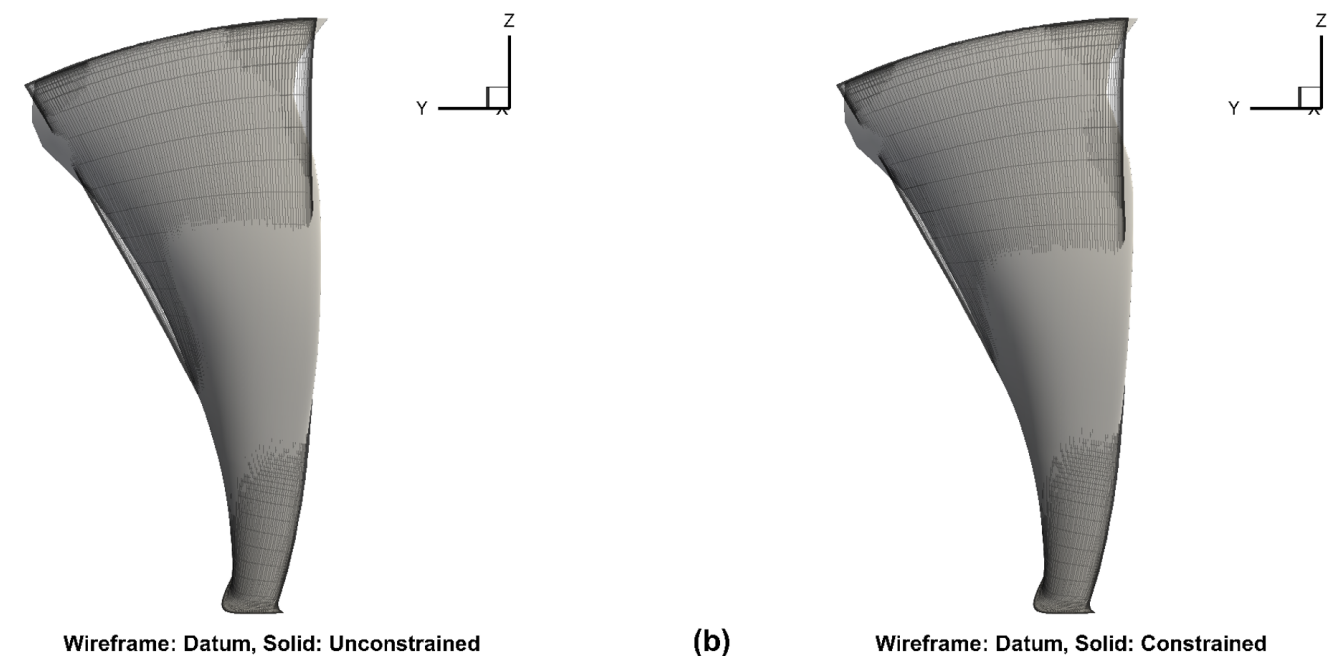

(a)

Wireframe: Datum, Solid: Unconstrained

(b)

Wireframe: Datum, Solid: Constrained

Fig. 15 Optimized blade shapes of the (a) unconstrained and (b) constrained aerodynamic optimizations. 


\section{Aeroacoustic and Aerodynamic Optimization}

The previous two sections have shown that by monodisciplinary optimizations, significant improvements in the aeroacoustic and aerodynamic performance at the design point of interest cannot be accomplished simultaneously. Therefore, in this section, the composite function $F(\boldsymbol{d} ; \alpha)$ is minimized under the $P R$-constraint of Eq. (13) for a series of $\alpha$ values. The $P W L$ s and efficiencies of the two constrained optimized designs in Sec. IV.B and Sec. IV.Care used for the scalings of the objectives. The datum fan blade is used as the initial geometry for all the optimizations.

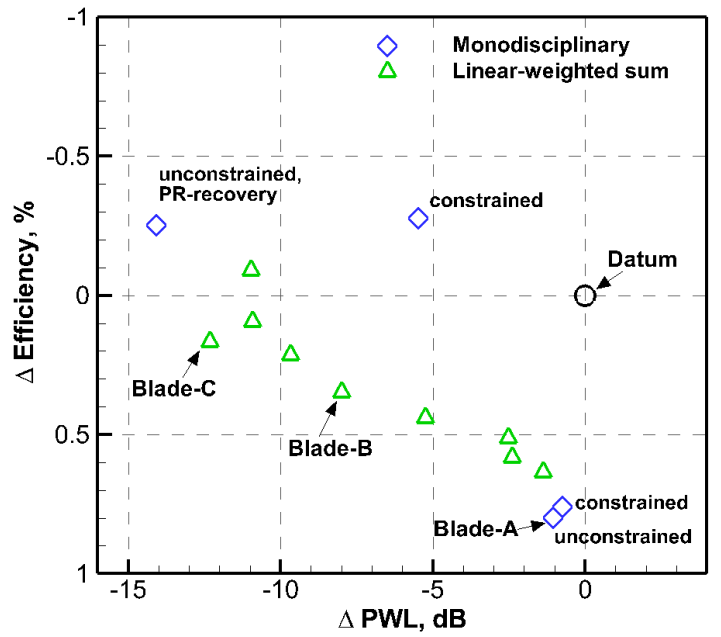

Fig. 16 Design solutions in the criterion space of the $P W L$ reduction and efficiency benefit.

The results are represented by delta symbols in the criterion space in Fig. 16 The datum fan blade is located at the origin and the two monodisciplinary optimization results are also given using diamond symbols. The use of the linear weighted sum of $P W L$ and efficiency successfully produces a set of design solutions that improve both the aeroacoustic and the aerodynamic performance of the fan and satisfy the aerodynamic constraints. It is interesting to note that, several solutions outperform the constrained, noise-optimized design not only in terms of efficiency but also in terms of noise. Overall, Fig. 16 might suggest that there exists a tradeoff between noise reduction and efficiency increase though not every solution satisfies the condition for Pareto optimality. Moving from the right to the left along the obtained solutions, a more significant $P W L$ reduction can be achieved as the efficiency benefit declines. In the meantime, by viewing how the blade shape changes, it is found that noise benefits at the design point are gradually increased following the increases in the leading-edge sweep amplitudes. So far, the effectiveness of the adjoint-based MOO approach for low-noise, transonic fan blade design has been demonstrated. 


\section{E. Analysis and Discussion}

Three design solutions, which are labelled as Blade-A, Blade-B, and Blade-C in Fig. 16, are selected for further analysis. First, efficiency spanwise profiles of the datum blade and Blade-A at the design point in Fig. 17]indicate that the biggest increase occurs at $95 \%$ span. Figure 18 then shows how the flow field at this section is improved by Blade-A. The main passage shock can be seen to change from being swallowed to being expelled. This movement not only eliminates the entropy generation across the swallowed, normal passage shock on the pressure side from $15 \%$ to $20 \%$ chord, but more apparently, it reduces the shock-induced separation on the suction side as well as the blade wake. Accordingly, the fan efficiency is improved.

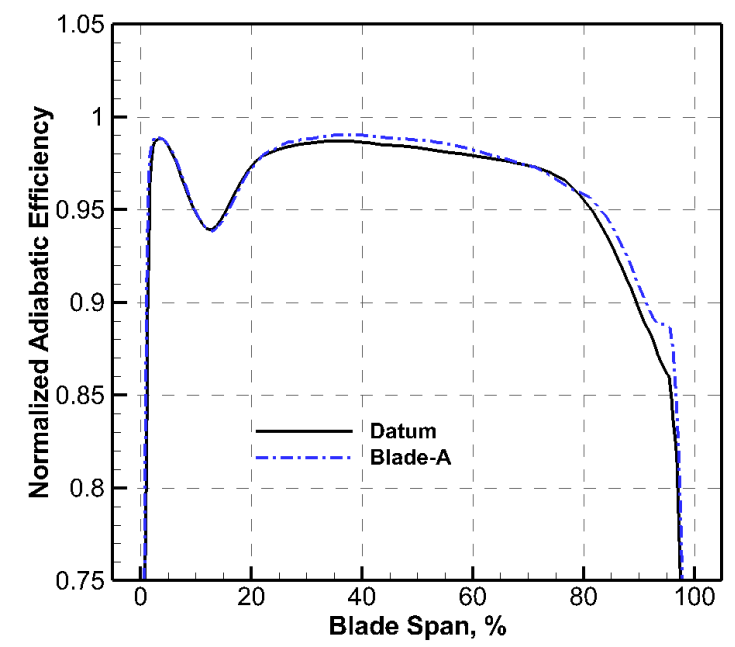

Fig. 17 Efficiency spanwise profiles of the datum blade and Blade-A at the design point.
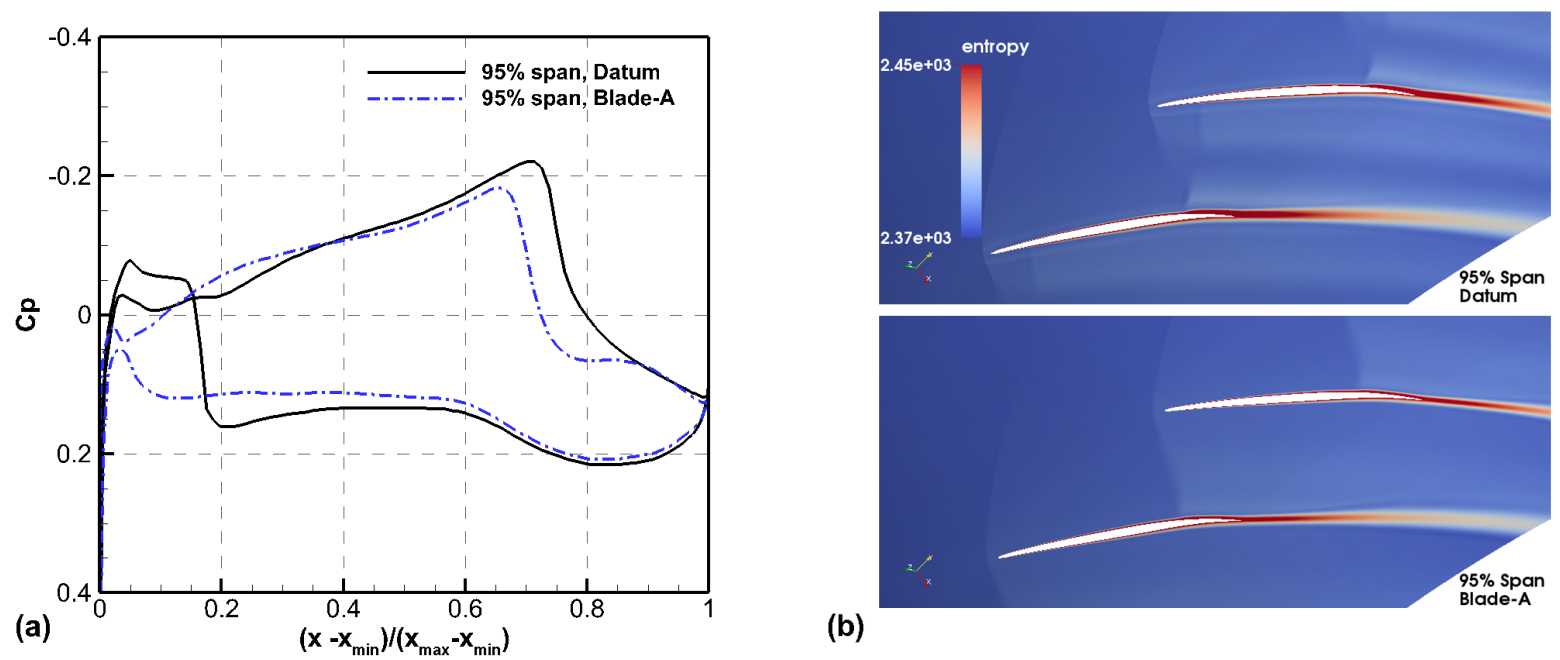

(b)

Fig. 18 (a) Blade loading distributions and (b) the entropy fields of the datum blade and Blade-A at 95\% span. 


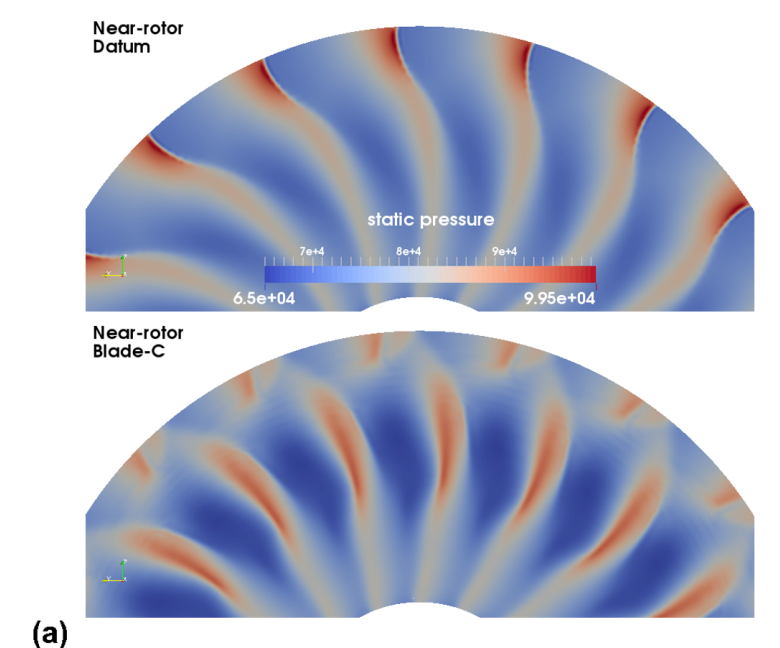

(b)

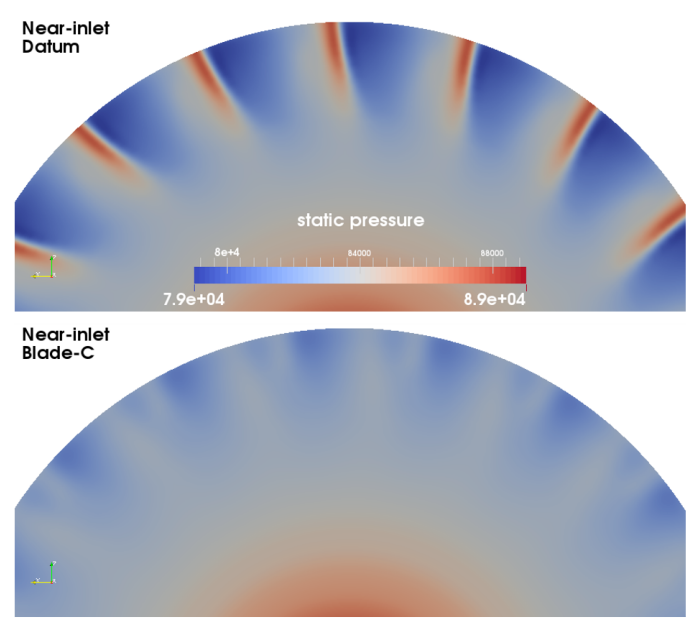

Fig. 19 Static pressure contours of the datum blade and Blade-C on (a) a near-rotor axial plane and (b) a near-inlet axial plane.
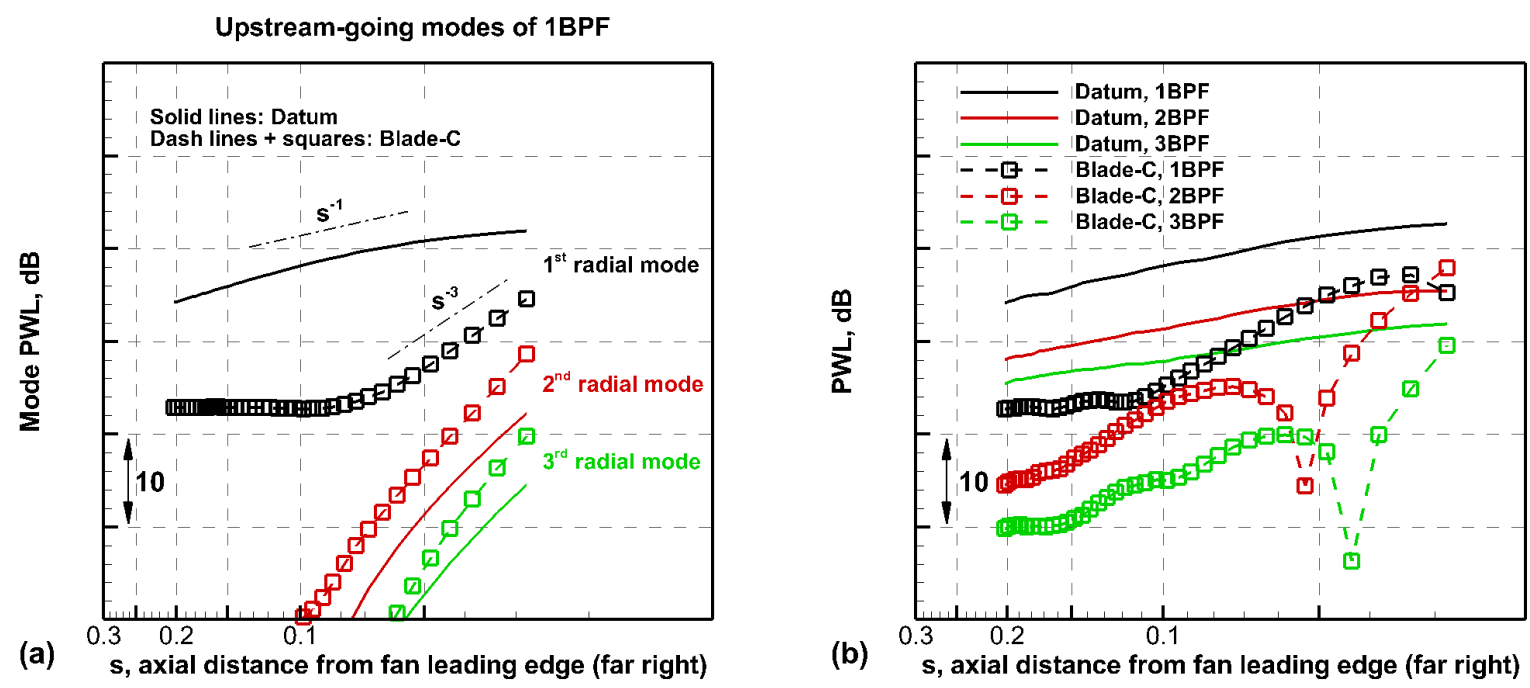

Fig. 20 Axial distribution of (a) mode $P W L$ s of the first three upstream-going radial modes at 1BPF and (b) $P W L s$ of the first three BPF harmonics for the datum blade and Blade-C at the design point.

The flow and acoustic fields of Blade-C at the design point are analyzed and compared with that of the datum blade to understand the mechanisms responsible for noise reduction. Acoustic results are obtained by the wavesplitting approach [14, 15]. Note that Blade-C has the same compound leading-edge sweep pattern as described in Sec.IV.B. Figure 19 shows that the near-rotor shock in each periodic domain is changed into a dual shock structure by the compound leading-edge sweep, and as a result, the near-inlet shock is almost eliminated. It can be inferred from Fig. 19.a) that the near-rotor perturbation field of the datum blade is almost entirely contributed by the first-order radial mode, whereas 
high-order radial modes, at least the second-order, are necessities to form the dual shock structure for Blade-C. Wilson et al. [3] pointed out that mismatching the shock field from the propagating duct modes while in favour of the cut-off modes was the noise reduction mechanism of the compound leading-edge sweep. This can be confirmed by Fig. 20.a), which shows the $P W L$ distribution of the first three upstream-going radial modes at 1BPF for the two blades. Only the first-order radial mode is cut-on at 1BPF for both cases. For the datum blade, the first-order radial mode closely follows the $s^{-1}$ near-field decay behaviour of the total sound power generated by shock waves [32] and has much higher $P W L$ s (at least $20 \mathrm{~dB}$ ) compared with high-order, cut-off modes. This means a strong coupling between the first-order radial mode and the shock field across the whole upstream region for the datum blade. However, for Blade-C, high-order radial modes possess more energy, especially in the near-rotor region. The initial faster decay of the first-order radial mode for Blade-C might show evidence of acoustic energy transferring to high-order radial modes, and then the first-order radial mode is seen to dominate the acoustic field while propagating linearly. Furthermore, not only the enhanced coupling between the near-rotor shock structure and high-order radial modes but also the enhanced coupling between the near-rotor shock structure and high-order circumferential harmonics, achieved by Blade-C, are found to be responsible for noise reduction. Figure 20 b) clearly shows that $2 \mathrm{BPF}$ takes over from $1 \mathrm{BPF}$ to become dominant at the very near-rotor location of Blade-C, while the whole upstream acoustic field of the datum blade is dominated by $1 \mathrm{BPF}$. The singular behaviour of the $P W L$ distribution of $2 \mathrm{BPF}$, as well as $3 \mathrm{BPF}$ for Blade-C, indicates a change of direction of the acoustic energy flow. At the near-rotor region, acoustic energy flows of $2 \mathrm{BPF}$ and $3 \mathrm{BPF}$ for Blade-C are in fact going downstream. This would be beneficial to the reduction of the total $P W L$ at the fan inlet.

Figure 21 compares the noise performance map and the rotor characteristics of the datum blade and the three selected blades to examine the off-design performance. Noise performance results show that throttling along a speed line would generally increase the total $P W L$ at the inlet. This trend is due to the forward movement of the shock structure as a result of throttling. Overall, it is encouraging that the improvements of the aeroacoustic and aerodynamic performance are realized, though not consistently, for all three blades across the whole mass flow range. Noise reduction is seen to decrease as moving from the choke point to the near-stall point for each design. The efficiency curves of Blade-C and Blade-B are found, perhaps unexpectedly, to be higher than that of Blade-A in a wide range below the peak efficiency points, whereas the efficiency benefits achieved by Blade-A are quite local around the design point. The above analysis suggests that multi-point optimizations might be necessary to obtain more robust designs of low-noise, high-efficiency fan blades.

Lastly, full-annulus CFD simulations for buzz-saw noise prediction are carried out at the design point to evaluate the actual noise reduction effect of Blade-C on other tones in addition to the BPF harmonics. The small manufacturing differences between the fan blades in reality are mimicked by introducing stagger angle variations at the tip of each blade and then linearly varying to zeroes at the blade hub [13]. An extra mesh block with axially-stretched grids is added in front of the original computational domain to further reduce numerical reflections. The predicted $P W L$ engine 

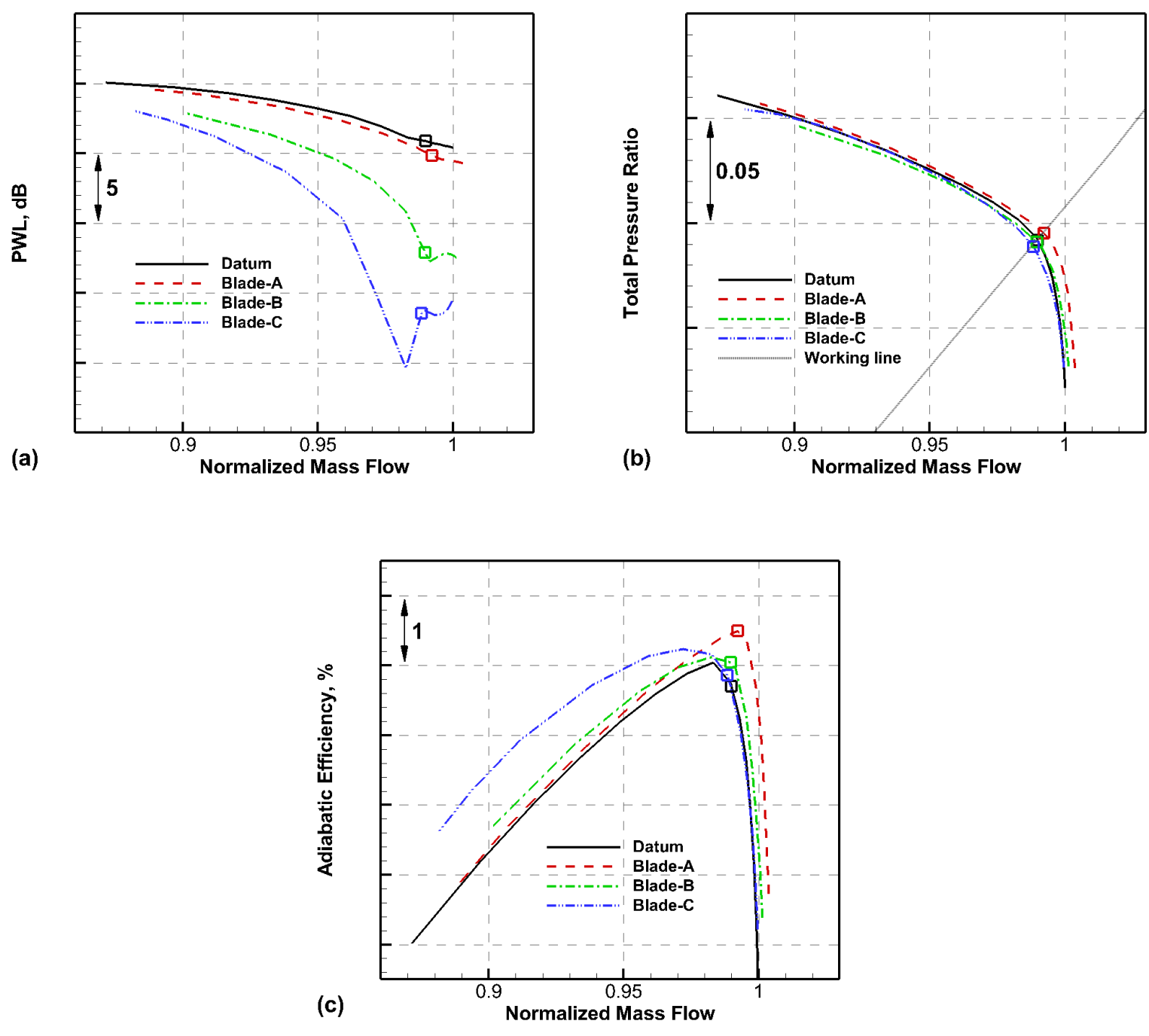

Fig. 21 Performance comparisons of (a) $P W L$, (b) total pressure ratio, and (c) adiabatic efficiency between the datum blade, Blade-A, Blade-B, and Blade-C across the operating range. (design point represented by squares)

order spectra at the fan inlet for Blade-C and the datum blade are shown in Fig. 22 All the rotor-alone tones up to 3BPF except the first three engine orders are reduced significantly by the present optimization.

\section{Conclusion}

In this paper, aeroacoustic adjoint analysis for the rotor-alone tone noise of transonic fans has been developed based on the discrete steady adjoint approach, thanks to the "rotor-locked" nature of the shock-associated tone noise. It has been applied together with aerodynamic adjoint simulations to establish an adjoint-based MOO process for low-noise, high-efficiency transonic fan blade design. An industrial transonic fan has been optimized using the adjoint-based MOO process to improve its aeroacoustic and aerodynamic performance, and the necessity of adopting the MOO approach has 


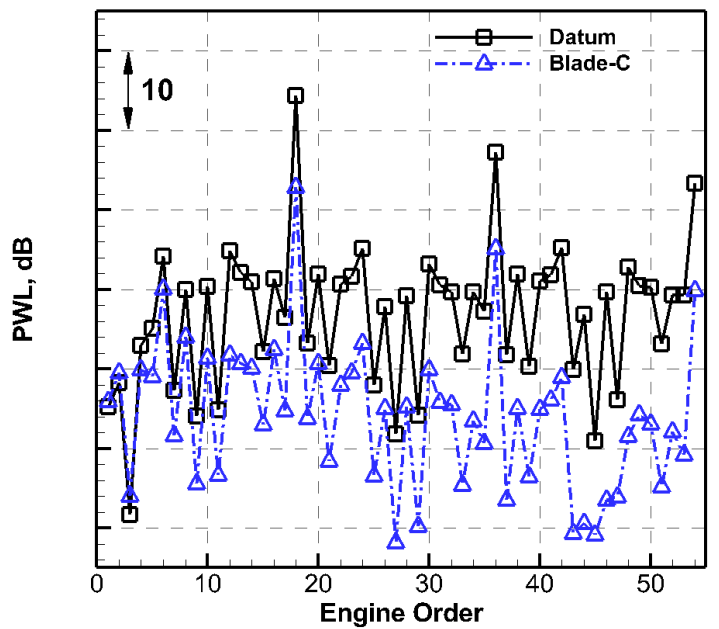

Fig. 22 Fan inlet $P W L$ engine order spectra of the datum blade and Blade-C at the design point.

been demonstrated by comparisons with monodisciplinary optimizations. Significant noise reductions and appreciable efficiency benefits are achieved at the design point of the transonic fan via the present optimizations.

A compound leading-edge sweep pattern has been generated and found responsible for the shock-associated tone noise reduction. It enhances the coupling between the shock field and high-order duct modes (both radial and circumferential) so that much less acoustic energy can propagate to the upstream far-field.

Design solutions obtained by the adjoint-based, aeroacoustic and aerodynamic optimizations have shown better performance across the whole mass flow range compared with the datum blade, although noise reduction decreases as throttling along the speed line. Besides, full-annulus analyses have demonstrated the effectiveness of the optimized blade using the definition of noise objective based on BPF harmonics to widely reducing the rotor-alone tones for transonic fans.

Further efforts could be made to conduct multi-point design and to include mechanical analysis in the MDO process for more robust and realistic fan blade designs. Other geometry parameterization techniques, e.g. the free-form deformation, could be explored using the adjoint-based approach to discover new blade shaping. The current adjoint analysis could also be combined with mode-matching techniques and surface integral methods to develop adjoint-based, aeroacoustic and aerodynamic shape design capabilities for coupled intake and fan systems.

\section{Funding Sources}

This work is funded by the MADELEINE project from the European Union's Horizon 2020 research and innovation programme under grant agreement No. 769025. 


\section{Acknowledgments}

The authors acknowledge Rolls-Royce's support for the computing facility and software used in this work.

\section{References}

[1] Luo, C., Song, L., Li, J., and Feng, Z., "A Study on Multidisciplinary Optimization of an Axial Compressor Blade Based on Evolutionary Algorithms," ASME Journal of Turbomachinery, Vol. 134, No. 5, 2012, p. 054501. https://doi.org/10.1115/1. 4003817

[2] Baert, L., Beaucaire, P., Leborgne, M., Sainvitu, C., and Lepot, I., "Tackling Highly Constrained Design Problems: Efficient Optimisation of a Highly Loaded Transonic Compressor," Proceedings of the ASME Turbo Expo 2017: Turbomachinery Technical Conference and Exposition, Turbomachinery, Vol. 2C, ASME, Charlotte, North Carolina, 2017. https://doi.org/10.1115/GT201764610

[3] Wilson, A., Stieger, R., Coupland, J., Smith, N., and Humphreys, N., "Multi-Disciplinary Optimisation of a Transonic Fan for Low Tone Noise," 17th AIAA/CEAS Aeroacoustics Conference (32nd AIAA Aeroacoustics Conference), AIAA, Portland, Oregon, 2011. https://doi.org/10.2514/6.2011-2950

[4] Kim, J. H., Ovgor, B., Cha, K. H., Kim, J. H., Lee, S., and Kim, K. Y., "Optimization of the Aerodynamic and Aeroacoustic Performance of an Axial-Flow Fan," AIAA Journal, Vol. 52, No. 9, 2014, pp. 2032-2044. https://doi.org/10.2514/1.J052754

[5] Morfey, C. L., and Fisher, M. J., "Shock-Wave Radiation from a Supersonic Ducted Rotor," The Aeronautical Journal, Vol. 74, No. 715, 1970, pp. 579-585. https://doi.org/10.1017/S0001924000049095

[6] Li, Z., and Zheng, X., "Review of Design Optimization Methods for Turbomachinery Aerodynamics," Progress in Aerospace Sciences, Vol. 93, 2017, pp. 1-23. https://doi.org/10.1016/j.paerosci.2017.05.003.

[7] Jameson, A., “Aerodynamic Design via Control Theory,” Journal of Scientific Computing, Vol. 3, 1988, pp. $233-260$. https://doi.org/10.1007/BF01061285

[8] Giles, M. B., and Pierce, N. A., “An Introduction to the Adjoint Approach to Design,” Flow, Turbulence and Combustion, Vol. 65, 2000, pp. 393-415. https://doi.org/10.1023/A:1011430410075

[9] Giles, M. B., Duta, M. C., Muller, J. D., and Pierce, N. A., "Algorithm Developments for Discrete Adjoint Methods," AIAA Journal, Vol. 41, No. 2, 2003, pp. 198-205. https://doi.org/10.2514/2.1961

[10] Giles, M. B., Ghate, D., and Duta, M. C., "Using Automatic Differentiation for Adjoint CFD Code Development," Recent Trends in Aerospace Design and Optimization, edited by B. Uthup, S. Koruthu, R. K. Sharma, and P. Priyadarshi, Tata McGraw-Hill, New Delhi, 2006, pp. 426-434.

[11] Duta, M. C., Shahpar, S., and Giles, M. B., “Turbomachinery Design Optimization Using Automatic Differentiated Adjoint Code," Proceedings of the ASME Turbo Expo 2007: Power for Land, Sea, and Air, Vol. 6: Turbo Expo 2007, Parts A and B, ASME, Montreal, 2007, pp. 1435-1444. https://doi.org/10.1115/GT2007-28329 
[12] Luo, J., Xiong, J., Liu, F., and McBean, I., “Three-Dimensional Aerodynamic Design Optimization of a Turbine Blade by Using an Adjoint Method," ASME Journal of Turbomachinery, Vol. 133, No. 1, 2011, p. 011026. https://doi.org/10.1115/1.4001166

[13] Coupland, J., Wilson, A. G., Pollard, N., Ullenberg, S., Breard, C., and Diamond, J., "Demonstration of a CFD-CAA Methodology to predict Buzz-Saw Noise Propagation to the Aircraft," 13th AIAA/CEAS Aeroacoustics Conference (28th AIAA Aeroacoustics Conference), AIAA, Rome, 2007. https://doi.org/10.2514/6.2007-3517

[14] Wilson, A. G., "A Method for Deriving Tone Noise Information from CFD Calculations on the Aero-Engine Fan Stage," NATO RTO-AVT Symposium on Developments in Computational Aero- and Hydro-Acosutics, Manchester, 2001.

[15] Moinier, P., and B, G. M., "Eigenmode Analysis for Turbomachinery Applications," AIAA Journal of Propulsion and Power, Vol. 21, No. 6, 2005, pp. 973-978. https://doi.org/10.2514/1.11000

[16] Daroukh, M., Moreau, S., Gourdain, N., Boussuge, J. F., and Sensiau, C., "Tonal Noise Prediction of a Modern Turbofan Engine with Large Upstream and Downstream Distortion,” ASME Journal of Turbomachinery, Vol. 141, No. 2, 2019 , p. 021010. https://doi.org/10.1115/1.4042163.

[17] Morfey, C. L., “Acoustic Energy in Non-Uniform Flows,” Journal of Sound and Vibration, Vol. 14, No. 2, 1971 , pp. 159-170. https://doi.org/10.1016/0022-460X(71)90381-6

[18] Lapworth, L., "Hydra-CFD : A Framework for Collaborative CFD Development," International Conference on Scientific and Engineering Computation (IC-SEC), Singapore, 2004.

[19] Seshadri, P., Parks, G. T., and Shahpar, S., "Leakage Uncertainties in Compressors: The Case of Rotor 37," AIAA Journal of Propulsion and Power, Vol. 31, No. 1, 2015, pp. 456-466. https://doi.org/10.2514/1.B35039

[20] Shahpar, S., and Lapworth, L., "PADRAM: Parametric Design and Rapid Meshing System for Turbomachinery Optimisation," Proceedings of the ASME Turbo Expo 2003, collocated with the 2003 International Joint Power Generation Conference, Vol. 6: Turbo Expo 2003, Parts A and B, ASME, Atlanta, Georgia, 2003, pp. 579-590. https://doi.org/10.1115/GT2003-38698.

[21] Maxwell, R. D., “MFR Build 2 - Vital Fan Rig Test Report,” Rolls-Royce Private Data, Rep. No. RCR 91463, Rolls-Royce plc., 2011.

[22] Denton, J. D., "The 1993 IGTI Scholar Lecture: Loss Mechanisms in Turbomachines," ASME Journal of Turbomachinery, Vol. 115, No. 4, 1993, pp. 621-656. https://doi.org/10.1115/1.2929299

[23] Deb, K., Pratap, A., Agarwal, S., and Meyarivan, T., “A Fast and Elitist Multiobjective Genetic Algorithm: NSGA-II,” IEEE Transactions on Evolutionary Computation, Vol. 6, No. 2, 2002, pp. 182-197. https://doi.org/10.1109/4235.996017.

[24] Sasaki, D., and Obayashi, S., "Efficient Search for Trade-Offs by Adaptive Range Multi-Objective Genetic Algorithms," Journal of Aerospace Computing, Information, and Communication, Vol. 2, No. 1, 2005, pp. 44-64. https://doi.org/10.2514/1.12909

[25] Marler, R. T., and Arora, J. S., “The Weighted Sum Method for Multi-Objective Optimization: New Insights,” Structural and Multidisciplinary Optimization, Vol. 41, No. 6, 2010, pp. 853-862. https://doi.org/10.1007/s00158-009-0460-7 
[26] Mavrotas, G., "Effective Implementation of the $\varepsilon$-Constraint Method in Multi-Objective Mathematical Programming Problems," Applied Mathematics and Computation, Vol. 213, No. 2, 2009, pp. 455-465. https://doi.org/10.1016/j.amc.2009.03.037

[27] Das, I., and Dennis, J. E., "Normal-boundary Intersection: A New Method for Generating the Pareto Surface in Nonlinear Multicriteria Optimization Problems," SIAM Journal on Optimization, Vol. 8, No. 3, 1998, pp. 631-657. https://doi.org/10. $1137 / \mathrm{S} 1052623496307510$

[28] Kraft, D., “A Software Package for Sequential Quadratic Programming,” Tech. Rep. DFVLR-FB-88-28, DLR German Aerospace Center - Institute for Flight Mechanics, Koln, Germany, 1988.

[29] Bliss, D., Hayden, R., Murray, B., and Schwaar, P., "Design Considerations for a Novel Low Source Noise Transonic Fan Stage," 3rd Aeroacoustics Conference, AIAA, Palo Alto, CA, 1976. https://doi.org/10.2514/6.1976-577

[30] Hawkings, D., "Multiple Tone Generation by Transonic Compressors," Journal of Sound and Vibration, Vol. 17, No. 2, 1971, pp. 241-250. https://doi.org/10.1016/0022-460X(71)90458-5.

[31] McAlpine, A., and Fisher, M. J., "On the Prediction of "Buzz-saw" Noise in Aero-engine Inlet Ducts," Journal of Sound and Vibration, Vol. 248, No. 1, 2001, pp. 123-149. https://doi.org/10.1006/jsvi.2001.3770

[32] Fink, M. R., "Shock Wave Behavior in Transonic Compressor Noise Generation,” ASME Journal of Engineering for Power, Vol. 93, No. 4, 1971, pp. 397-403. https://doi.org/10.1115/1.3445598 